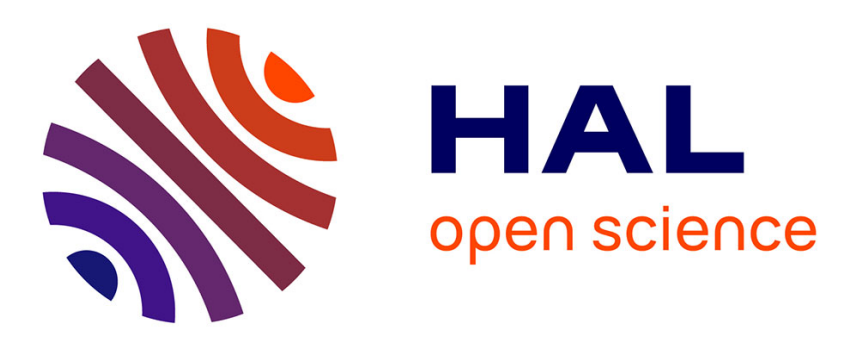

\title{
Attitude Estimation for Indoor Navigation and Augmented Reality with Smartphones
}

\author{
Thibaud Michel, Pierre Genevès, Hassen Fourati, Nabil Layaïda
}

\section{To cite this version:}

Thibaud Michel, Pierre Genevès, Hassen Fourati, Nabil Layaïda. Attitude Estimation for Indoor Navigation and Augmented Reality with Smartphones. Pervasive and Mobile Computing, 2018, 46, pp.96-121. 10.1016/j.pmcj.2018.03.004 . hal-01650142v2

\section{HAL Id: hal-01650142 \\ https://hal.inria.fr/hal-01650142v2}

Submitted on 2 Mar 2018

HAL is a multi-disciplinary open access archive for the deposit and dissemination of scientific research documents, whether they are published or not. The documents may come from teaching and research institutions in France or abroad, or from public or private research centers.
L'archive ouverte pluridisciplinaire HAL, est destinée au dépôt et à la diffusion de documents scientifiques de niveau recherche, publiés ou non, émanant des établissements d'enseignement et de recherche français ou étrangers, des laboratoires publics ou privés. 


\title{
Attitude Estimation for Indoor Navigation and Augmented Reality with Smartphones
}

\author{
Thibaud Michel ${ }^{\mathrm{a}, \mathrm{b}}$, Pierre Genevès ${ }^{\mathrm{a}}$, Hassen Fourati ${ }^{\mathrm{b}}$, Nabil Layaïda ${ }^{\mathrm{a}}$ \\ ${ }^{a}$ LIG, Inria, Univ. Grenoble Alpes, CNRS, Grenoble, France \\ ${ }^{b}$ Univ. Grenoble Alpes, CNRS, Inria, Grenoble INP, GIPSA-Lab, 38000 Grenoble, France
}

\begin{abstract}
We investigate the precision of attitude estimation algorithms in the particular context of pedestrian navigation with commodity smartphones and their inertial/magnetic sensors. We report on an extensive comparison and experimental analysis of existing algorithms. We focus on typical motions of smartphones when carried by pedestrians. We use a precise ground truth obtained from a motion capture system. We test state-of-the-art and built-in attitude estimation techniques with several smartphones, in the presence of magnetic perturbations typically found in buildings. We discuss the obtained results, analyze advantages and limits of current technologies for attitude estimation in this context. Furthermore, we propose a new technique for limiting the impact of magnetic perturbations with any attitude estimation algorithm used in this context. We show how our technique compares and improves over previous works. A particular attention was paid to the study of attitude estimation in the context of augmented reality motions when using smartphones.
\end{abstract}

Keywords: Attitude Estimation, Smartphone, Inertial Sensors, Augmented Reality Motions, Magnetic Field, Perturbations, Benchmark.

\section{Introduction}

Pervasive applications on smartphones increasingly rely on techniques for estimating attitude. Attitude is the orientation of the smartphone with respect to Earth's local frame [1]. Augmented Reality applications [2, 3, 4], pedestrian dead-reckoning systems for indoor-localisation [5], and photo sphere creations and previews [6] constitute examples in which precision and stability of attitude estimation matter. For example, in the Pedestrian Dead Reckoning (PDR) process, the attitude estimation is used to determine a user direction. If this information is coupled with a step detection algorithm [7], the relative position of a user can be determined (Fig. 1]. Augmented Reality (AR) is another example where the reliability of attitude estimation is important. AR is a live view of a real world environment where virtual objects are shown over the camera image of a hand-held device. Geo AR [8] (or Gravimetric AR) is an AR method which

Email addresses: thibaud.micheleinria.fr(Thibaud Michel), pierre.geneves@cnrs.fr (Pierre Genevès), hassen.fourati@gipsa-lab.fr(Hassen Fourati), nabil. layaida@inria.fr (Nabil Layaïda) 


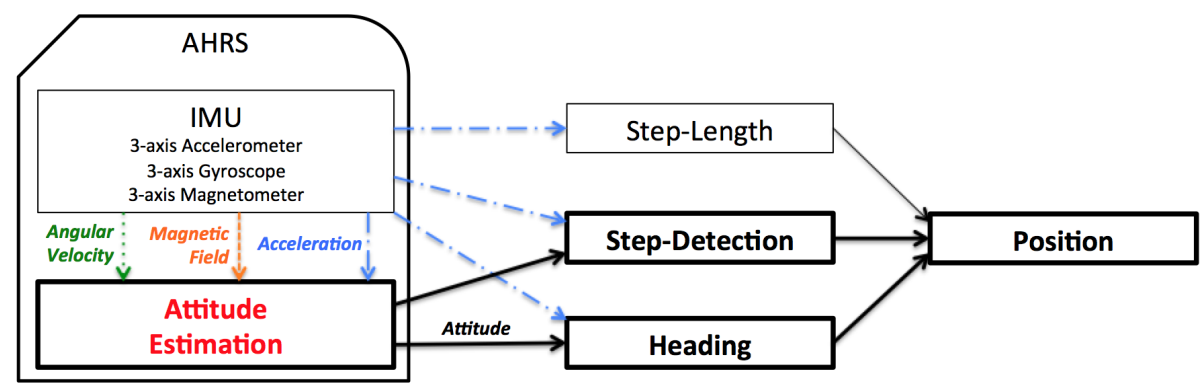

Figure 1: Pedestrian Dead Reckoning (PDR) process.

relies exclusively on device position and attitude (Fig. 2). This technique does not use image processing. GPS, Wifi, Bluetooth or any kind of location sensors can be used to determine a device position. Precision of attitude estimation is crucial in Geo AR, as virtual objects should be displayed at the right place. Stability of attitude estimation is crucial as well, since movements of virtual objects should be perceived as following camera movements.

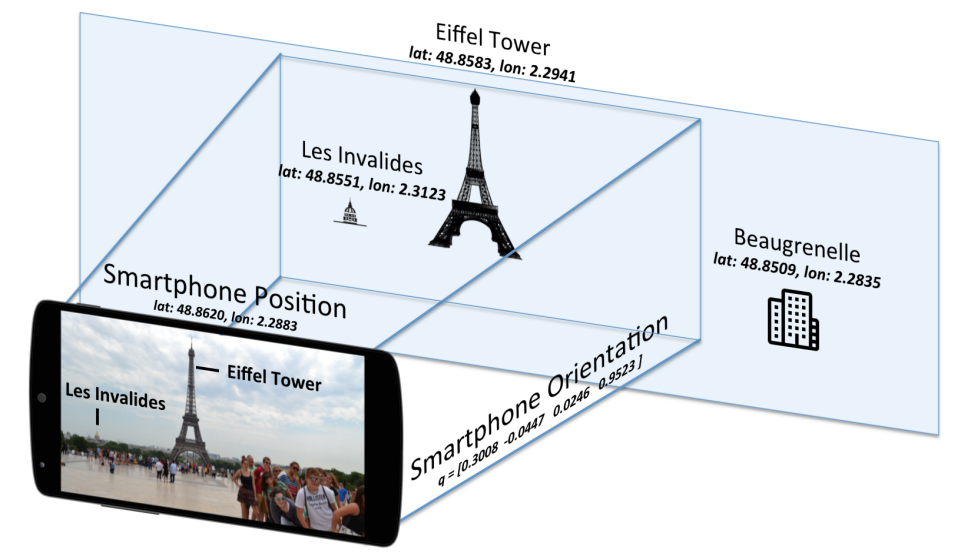

Figure 2: Geo Augmented Reality (Geo AR) approach.

Modern smartphones embed sensors such as accelerometer, gyroscope, and magnetometer which make it possible to leverage existing attitude estimation algorithms. Such algorithms have been extensively investigated in various domains such as: robotics [9], aerospace [10], unmanned aerial vehicles [11], bio-logging [12] and indoor positioning [5]. However, the particular context of smartphones carried by pedestrians brings new challenges due to singular accelerations and magnetic perturbations, which sometimes invalidate the basic hypotheses that underly state-ofthe-art attitude estimation algorithms. In particular, the absence of model describing the smartphone motions (preventing control), and the presence and variations of magnetic perturbations during the estimation phase, both introduce additional difficulties.

Contribution. We investigate the precision of attitude estimation algorithms in the context of commodity smartphones carried by pedestrians with a specific focus on AR. We consider eight 
typical motions (such as texting, phoning, running, etc.) with various impacts on external accelerations, as well as the presence/absence of magnetic perturbations typically found in indoor environments. We systematically analyze, compare and evaluate eight state-of-the-art algorithms (and their variants). We precisely quantify the attitude estimation error obtained with each technique, owing to the use of a precise ground truth obtained with a motion capture system. We make our benchmark available 1 and pay attention to the reproducibility of results. We analyze and discuss the obtained results and report on conclusions. We present a new technique which helps in improving precision by limiting the effect of magnetic perturbations with all considered algorithms. We also adapted this new technique with the best practices learned from the literature to obtain a precise and stable filter for AR.

Outline of the paper. We first introduce required preliminaries in Section 2 Then, we review the closest related works in Section 3 . We present the considered algorithms in Section 4 , our experimental protocol in Section 5, and obtained results in Section 6 We propose our new technique to limit the impact of magnetic perturbations in Section 7 . Finally, we report on attitude estimation for AR in Section 8 before concluding in Section 9

\section{Background for attitude estimation}

\subsection{Sensors measurements and calibration}

The sensors configuration of a smartphone is composed of a triad of MEMS (Micro-ElectroMechanical Systems) sensors consisting of a 3-axis gyroscope, a 3-axis accelerometer and a 3 -axis magnetometer. The outputs of these low-cost sensors are imprecise as they suffer from several problems: noise, bias, full scale range, axes misalignment, axes non-orthogonality and temperature variations. Sensors models are described below.

\subsubsection{Gyroscope}

The 3-axis gyroscope measures the angular velocity of the smartphone in $\mathrm{rad}_{\mathrm{s}} \mathrm{s}^{-1}$ : gyr $=$ $\left[\begin{array}{lll}\operatorname{gyr}_{x} & \operatorname{gyr}_{y} & \operatorname{gyr}_{z}\end{array}\right]^{T}$. The widely used continuous time model for a gyroscope can be written as:

$$
\operatorname{gyr}=\operatorname{gyr}_{r}+\operatorname{gyr}_{b}+\operatorname{gyr}_{n},
$$

where:

gyr is the angular rate measured by the gyroscope.

$\operatorname{gyr}_{r}$ is the true angular rate.

$\operatorname{gyr}_{b}$ is the gyroscope bias.

$\operatorname{gyr}_{n}$ is the gyroscope noise.

A gyroscope during a static phase should provide an angular velocity of 0 for each axis. Due to the poor quality of sensors, measurements show a small bias. This bias $\left(\mathrm{gyr}_{b}\right)$ can be detected during static phases and is then subtracted from measurements during online phase.

$\sqrt[1]{\text { http://tyrex.inria.fr/mobile/benchmarks-attitude }}$ 


\subsubsection{Accelerometer}

The 3-axis accelerometer measures the acceleration of the smartphone, including the gravity and external acceleration in $m . s^{-2}:$ acc $=\left[\begin{array}{lll}\operatorname{acc}_{x} & \operatorname{acc}_{y} & \operatorname{acc}_{z}\end{array}\right]^{T}$. The continuous time model for an accelerometer can be written:

$\mathrm{acc}=\mathrm{acc}_{r}+\operatorname{acc}_{b}+\operatorname{acc}_{n}$,

where:

acc is the sum of the gravity and external acceleration of the body measured by the accelerometer (Eq. 3).

$\operatorname{acc}_{r}$ is the true sum of the gravity and external acceleration of the body.

$\operatorname{acc}_{b}$ is the accelerometer bias.

$\operatorname{acc}_{n}$ is the accelerometer noise.

Gravity is the force of attraction by which terrestrial body tends to fall toward the center of the earth and external accelerations are all others accelerations applied on the body:

$$
\text { acc }=\text { gravity }+\operatorname{acc}^{e x t} .
$$

An accelerometer during a static phase provides a magnitude of acceleration close to $g$, where $g$ is the acceleration due to the gravity at the Earth's surface $\left(g \approx 9.8 \mathrm{~m} . \mathrm{s}^{-2}\right)$. In [13], authors provide an accelerometer calibration algorithm based on a minimum of 9 static phases. This calibration allows to remove the bias and misalignment by normalizing the acceleration vector in multiple smartphone orientations.

\subsubsection{Magnetometer}

The 3-axis magnetometer measures the magnetic field in the smartphone frame in micro-tesla $(\mu T): \operatorname{mag}=\left[\begin{array}{lll}\operatorname{mag}_{x} & \operatorname{mag}_{y} & \operatorname{mag}_{z}\end{array}\right]^{T}$. The continuous time model for a magnetometer can be written such as:

$$
\operatorname{mag}=\operatorname{mag}_{r}+\operatorname{mag}_{b}+\operatorname{mag}_{n}
$$

where:

mag is the sum of the Earth's magnetic field and other magnetic fields measured by the magnetometer (Eq. 5).

$\operatorname{mag}_{r}$ is the true sum of the Earth's magnetic field and other magnetic fields.

$\operatorname{mag}_{b}$ is the magnetometer bias.

$\operatorname{mag}_{n}$ is the magnetometer noise. 


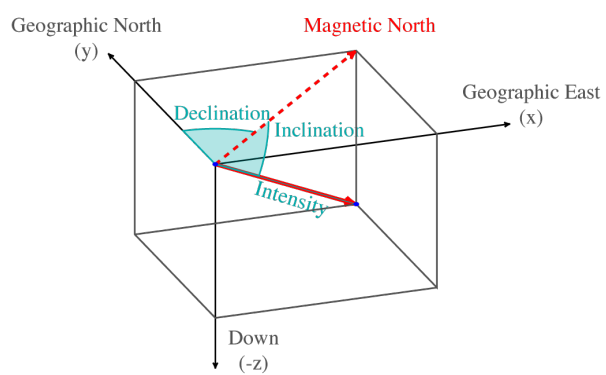

Figure 3: Earth's magnetic field representation

The Earth's magnetic field can be modeled by a dipole and follows basic laws of magnetic fields. At any location, the Earth's magnetic field can be represented by a three-dimensional vector and its intensity varies from $25 \mu T$ to $65 \mu T$. The National Geospatial-Intelligence Agency (NGA) and the United Kingdoms Defence Geographic Centre (DGC) provide a World Magnetic Model (WMM) [14] every 5-years. Declination is used to know the angle between the Magnetic North and Geographic North, while inclination and intensity are used to build the reference vector.

Unfortunately, the magnetometer does not measure only the Earth's magnetic field. Most of the time in indoor environments, we are in presence of magnetic dipoles which perturb the measure of Earth's magnetic field. These perturbations can be caused by electromagnetic devices (speakers, magnets), manmade structures (walls, floors) or other ferromagnetic objects like belts, keys, etc. For example, a smartphone speaker has a field of about $200 \mu T$ (4 times more than the Earth's magnetic field). The study found in [15] categorizes the environmental characteristics with respect to the magnetic deviations.

Earth's magnetic field is a vector pointing toward magnetic north and its magnitude is noted $F$. All other magnetic fields applied on the body are called magnetic perturbations and noted mag $^{\text {ext }}$ :

$$
\operatorname{mag}=\text { Earth's magnetic field }+ \text { mag }^{\text {ext }} .
$$

Magnetic perturbations are categorized in two groups: hard and soft iron distortions. Hard iron distortions are caused by ferromagnetic materials in the same frame than the smartphone (e.g. speaker for a smartphone). Soft iron distortions are caused by objects that produce a magnetic field (buildings walls, machines, heaters...) in a fixed frame. In a context free from magnetic interferences, hard and soft iron distortions can be partially corrected at the same time by normalizing the magnetic field vector in multiple smartphone orientations [16, 17]. In theory, due to soft iron distortions, when the device is moving or when the magnetic context changes, the calibration phase needs to be reprocessed.

\subsection{Attitude representation}

The smartphone attitude is determined when the axis orientation of the Smartphone-Frame $\mathrm{SF}\left(\mathrm{SF}_{x}, \mathrm{SF}_{y}, \mathrm{SF}_{z}\right)$ is specified with respect to the Earth-Frame $\mathrm{EF}\left(\mathrm{EF}_{x}, \mathrm{EF}_{y}, \mathrm{EF}_{z}\right)$ (or Local Tangent Plane (LTP)), see Fig.4 


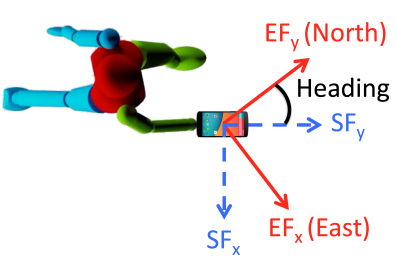

(a) Top View

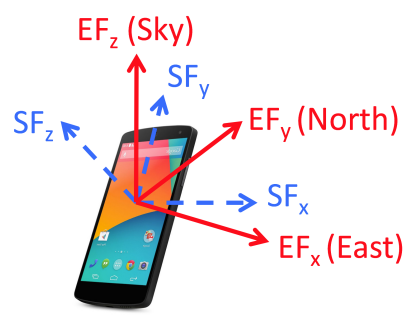

(b) Side View

Figure 4: The Smartphone-Frame SF (dashed line) and Earth-Frame EF (solid line).

The $\mathrm{SF}_{x}$-axis is horizontal and points to the right, the $\mathrm{SF}_{y}$-axis is vertical and points up and the $\mathrm{SF}_{z}$-axis points towards the outside of the front face of the screen. The $\mathrm{EF}_{y}$-axis points to the North. The $\mathrm{EF}_{z}$-axis points to the sky perpendicular to the reference ellipsoid and the $\mathrm{EF}_{x}$-axis completes the right-handed coordinate system, pointing East (ENU : East, North, Up). Another convention is often used by aerial vehicles called NED for North, East and Down.

Based on the literature, the attitude can be expressed with four different mathematical representations [18]. Euler angles (yaw, pitch, roll), rotation matrices, quaternions or axis/angle.

A unit-norm quaternion, which defines the rotation between $S F$ and $E F$, is defined by:

$$
q={ }_{S}^{E} q=\left[\begin{array}{llll}
q_{w} & q_{x} & q_{y} & q_{z}
\end{array}\right]^{T} \in \mathbb{R}^{4},
$$

where $q_{w}$ and $\left[\begin{array}{lll}q_{x} & q_{y} & q_{z}\end{array}\right]$ are respectively the scalar and the vector parts of the quaternion.

To express a vector $v=\left[\begin{array}{lll}v_{x} & v_{y} & v_{z}\end{array}\right]^{T}$ from EF to SF, Hamilton product [19] is used (Eq. (7)). Conversely, from EF to SF, Eq. (8) is used.

$$
\begin{aligned}
& { }^{S} v_{q}=q^{-1} \otimes{ }^{E} v_{q} \otimes q, \\
& { }^{E} v_{q}=q \otimes{ }^{S} v_{q} \otimes q^{-1},
\end{aligned}
$$

where $v_{q}$ is the quaternion form of $v$ (Eq. (9p)

$$
v_{q}=\left[\begin{array}{llll}
0 & v_{x} & v_{y} & v_{z}
\end{array}\right]^{T} .
$$

The well-known kinematic equation can be used to describe the variation of the attitude in term of quaternion:

$$
\dot{q}=\frac{1}{2} q \otimes \omega_{q},
$$

where $\omega_{q}$ is the quaternion form of angular velocity. More details about quaternion algebra can be found in [19].

Each representation has some drawbacks. In our context, Euler angles cannot be used due to the well-known gimbal-lock problem [20], when the device is in a pocket or held for phoning, the yaw angle can vary widely. Nevertheless, quaternions avoid the singularity problem, they provide basic primitives with cheap computation cost. All the algorithms that we have implemented in Java/Matlab and benchmarked in Section 6 use the quaternion algebra. A simple mathematical transformation between quaternions and Euler angles can be found in [20] for more intuitive rotations. 


\subsection{Attitude Estimation}

The problem of finding the optimal attitude estimation solution was formulated for the first time by Wahba in 1965 [1]. Wahba's problem seeks to find a rotation matrix between two coordinate systems from a set of vector observations (minimum two vectors known in a fixed frame and in a body frame). In our case, the two coordinate systems are the Smartphone Frame (SF) and the Earth Frame (EF) as shown in Fig. 4. A typical Inertial Measurement Unit (IMU) embedded in a smartphone can provide two vector observations expressed in two frames:

- acceleration in SF provided by an accelerometer noted ${ }^{S}$ acc and its projection in EF noted Eacc.

- magnetic field in SF provided by a magnetometer noted ${ }^{S}$ mag and its projection in EF noted ${ }^{E}$ mag.

These 2 observation vectors can be modeled as following:

$$
\begin{aligned}
& S_{\operatorname{acc}_{q}}=q^{-1} \otimes{ }^{E} \operatorname{acc}_{q} \otimes q, \\
& { }_{\operatorname{mag}_{q}}=q^{-1} \otimes{ }^{E} \operatorname{mag}_{q} \otimes q .
\end{aligned}
$$

If the smartphone is in static phase (not translating), acc ${ }^{e x t}=\left[\begin{array}{lll}0 & 0 & 0\end{array}\right]^{T}$ and

$$
E_{\text {acc }}=\left[\begin{array}{lll}
0 & 0 & g
\end{array}\right]^{T} .
$$

In absence of magnetic deviations, mag $^{e x t}=\left[\begin{array}{lll}0 & 0 & 0\end{array}\right]^{T}$ and

$$
{ }^{E} \operatorname{mag}=\left[\begin{array}{lll}
m_{x} & m_{y} & m_{z}
\end{array}\right]^{T},
$$

where $m_{x}, m_{y}$ and $m_{z}$ can be obtained using the WMM [14].

Figure 5 shows these two vectors: ${ }^{E}$ acc in blue and ${ }^{E}$ mag in green.

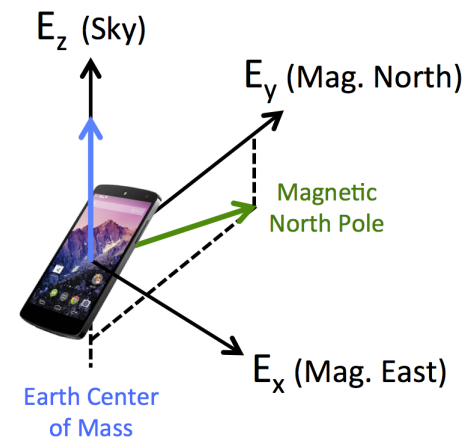

Figure 5: Reference vectors when the smartphone is static and in the absence of magnetic deviations.

In addition to accelerometer and magnetometer, the gyroscope is usually used to estimate variation of attitude. Unfortunately, the gyroscope bias leads after integration (Eq. (10) ) to an angular drift, increasing linearly over time. Since the use of only gyroscope is not enough for 
attitude estimation, accelerometer and magnetometer are used to get an absolute quaternion and compensate the drift. The crux in solving attitude estimation problem consists finally in combining inertial and magnetic sensor measurements in a relevant manner. Fig. 6illustrates the whole approach, where $K$ is the gain that allows to well merge data from accelerometer-magnetometer fusion and gyroscope integration. This gain is adjusted depending on sensors reliability.

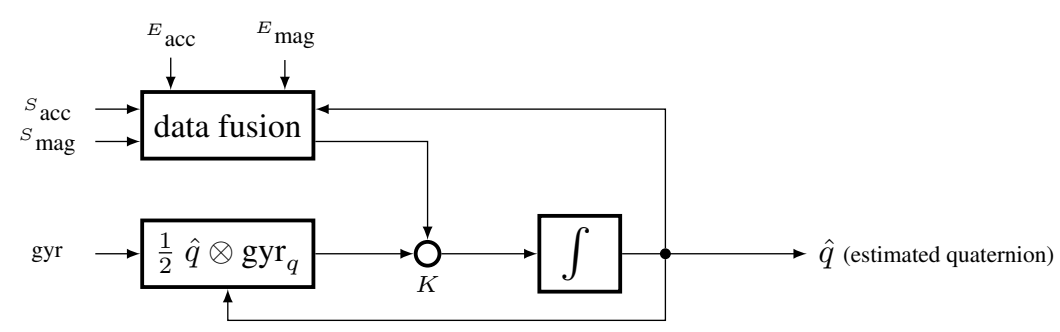

Figure 6: General approach for attitude estimation.

\section{Related Works}

Since 1965, a multitude of solutions have been proposed to resolve attitude estimation problem, such as TRIAD [21], QUaternion ESTimator (QUEST) [22], Singular Value decomposition method (SVD) [23], Kalman Filters (KF) [24, 25, 26, 27, 28], Extended Kalman Filters (EKF) [29, 30, 31, 32, 5], Unscented Kalman Filters (UKF) [33], Adaptive Kalman Filters (AKF) [34, 35], Particle Filters [36] and more recently Observers [12, 37, 38, 39]. A survey and an analysis of these methods can be found in [40]. In 2007, Crassidis et al. provide another survey with a focus on nonlinear attitude estimation methods. In this paper we further focus on algorithms that use measurements from the 3 sensors that are now commonly found on smartphones: gyroscopes, accelerometers and magnetometers, and attempt to leverage on these measurements to provide precise attitude estimation of smartphones carried by pedestrians.

Preliminary versions of this work were presented in [41] and [42] conferences. Compared to these earlier results, the present article mainly comprises three additional contributions: (i) more detailed descriptions on the considered motions and magnetic perturbations, in particular with a quantitative characterization; (ii) the overall study is extended with a specific focus on augmented reality applications; (iii) novel results concerning stability and precision balances achievable with each filter. We report on a systematic search of parameters adjustment with each filter to shed light on the feasibility envelope in terms of precision and stability.

Most algorithms developed so far rely on a common assumption: the external acceleration is negligible. However, when used in the context of smartphone carried by a pedestrian, this assumption is questionable (we have experimentally observed high external accelerations: see e.g. second column of Table 11. Specifically, the relation between ${ }^{S}$ acc and ${ }^{E}$ acc given by Eq. (11) is true only if no external acceleration is applied on the smartphone. Assumption of external acceleration is not a new problem, in [24, 25, 30, 27] authors propose to discard accelerometer measurements in the update phase of their KFs. They set values of covariance matrix to infinity when:

$$
\underbrace{\mid\|\|^{S} \text { acc }\|-\|^{E} \text { acc } \| \mid}_{\mu}>\gamma_{\text {acc }} .
$$


In [32] and [43], they explain how they adjust the covariance matrix in function of the left term of Eq. (15). In [34] and [35], authors use KF residual errors to detect external acceleration. The technique proposed in [34] needs time to let residual matrix converge in a static phase to identify bias before estimating external accelerations. Finally, in [5], Renaudin et al. only perform the update phase of their KF during periods considered as Quasi Static Field (QSF). During QSF, a low variance is given to measurements and ${ }^{E}$ acc is adjusted during these phases. To the best of our knowledge, the use of a detector à la (15) has not been investigated yet with an observer-based filter.

Most algorithms found in the literature do not consider magnetic perturbations. However, in the pedestrian context, the smartphone is often exposed to ferromagnetic objects, and this is known to yield a bad attitude estimation [15, 44]. Few papers are concerned with magnetic perturbations for attitude estimation on a smartphone carried by a pedestrian. In [39], authors consider the impact of magnetic perturbations on the North-East plane, abstracting over other possible impacts. In [24] and [30], authors set the covariance matrix of magnetic measurements to infinity when:

$$
\left|\left\|^{S} \operatorname{mag}\right\|-\left\|^{E} \operatorname{mag}\right\|\right|>\gamma_{\operatorname{mag}} .
$$

In [24], in addition to detector [16, Harada et al. use the following property to detect magnetic perturbations:

$$
\begin{aligned}
& \theta\left({ }^{S} \text { acc, }{ }^{S} \text { mag }\right)-\theta\left({ }^{E} \text { acc, },{ }^{E} \text { mag }\right)>\gamma_{\theta}, \\
& \text { where: } \theta\left(v_{1}, v_{2}\right)=\arccos \frac{v_{1} \cdot v_{2}}{\left\|v_{1}\right\| \cdot\left\|v_{2}\right\|} .
\end{aligned}
$$

Similarly to how external accelerations are treated, Renaudin et al. [5] use a QSF detector based on variance of measurements.

In the present paper, we develop a new technique for limiting the impact of magnetic perturbations on attitude estimation algorithms that are executed on smartphones carried by pedestrians. In addition, we conduct extensive practical experiments with several (and typical) motions of smartphones carried by a pedestrian, and show how our approach compares and improves over previous works in this context. To the best of our knowledge, our systematic comparison of attitude estimation algorithms is the first in this context. Our experiments include 126 datasets with several typical motions, several devices, realistic magnetic perturbations, and a fine-grained analysis.

\section{Selected Attitude Estimation Algorithms for Comparison}

We now review the state-of-the-art algorithms that we consider in our study. We have selected 8 filters from the literature which are representative of the different techniques developed for solving the attitude estimation based on IMU sensors. Our selection of algorithms can roughly be divided into two categories: those based on observers, and those based on KFs (with their EKF, UKF, and AKF variants). We summarize the main principles and objectives of each algorithm (see [41] for a more formal description of each algorithm using a common notation). For reproducibility purposes, we also indicate parameters that we used with each algorithm which we set in accordance with authors guidelines found in their papers. We also consider the "black-box algorithms" embedded in Android and iOS. The considered algorithms are the followings: 
Madgwick et al. [39]. This filter is a Gradient Descent (GD) based algorithm designed for pedestrian navigation. The authors propose to consider magnetic field deviations only on North-East plane using the following technique: ${ }^{E} \mathrm{mag}=\left[\begin{array}{lll}0 & m_{y} & m_{z}\end{array}\right]^{T}$, where $m_{y}=$ $\sqrt{h_{x}^{2}+h_{y}^{2}}, m_{z}=h_{z}$ and $h=\hat{q}^{-1} \otimes{ }^{S} \operatorname{mag} \otimes \hat{q}$. Madgwick is the common implementation of the filter, and MadgwickB the same with a gyro bias. Parameters: $\beta=0.08, \zeta=0.016$.

Martin et al. [38]. This filter is an observer with a new geometrical structure (invariant observer). The authors introduce new measurements based on the cross product of acceleration and magnetic field. Martin is the common implementation of the filter. Parameters: $l_{a}=0.7, l_{c}=0.1, l_{d}=0.01, n=0.01, o=0.01, k=10, \sigma=0.002$.

Mahony et al. [37]. This filter is a complementary filter designed for aerial vehicles. The main idea is to calculate the error by cross multiplying measured and estimated vectors. Mahony is the common implementation of the filter. MahonyB is the implementation which takes into account a gyro bias. Parameters: $\beta=1, \zeta=0.2$.

Fourati et al. [12]. This filter is a mix between a complementary filter algorithm and the Marquardt approach designed for bio-logging. Fourati is the common implementation of the filter. FouratiExtAcc is an extension which takes external accelerations into account using Eq. (15)). Parameters: $\beta=0.3, K_{a}=2$ and $K_{m}=1 . K_{a}=0$ when $\gamma_{\text {acc }}=0.5 \mathrm{~m} \cdot \mathrm{s}^{-2}$.

Choukroun et al. [26]. This filter provides a linearization of measurement equations. A KF is proposed and guarantees a global convergence. Choukroun is the common implementation of the filter.

Renaudin et al. [5]. This filter is an EKF designed for Pedestrian Dead Reckoning (PDR). In addition to Eq. (11) and Eq. [12), they use two others properties:

$$
\begin{aligned}
& a c c_{t+1}=q_{\omega}^{-1} \otimes a c c_{t} \otimes q_{\omega}, \\
& \operatorname{mag}_{t+1}=q_{\omega}^{-1} \otimes \operatorname{mag}_{t} \otimes q_{\omega},
\end{aligned}
$$

where $q_{\omega}$ is interpreted as a rotation between two successive epochs. Eq. 11], 12, 18, and (19) are applied only during QSF periods. The detector for QSF works by analyzing variance of acceleration and magnetic field measurements on a small window $(\approx 0.2 s)$. This filter has to be initialized $(\approx 5 s$ at the beginning) without external accelerations and magnetic perturbations (mostly outside). Renaudin is the common implementation of the filter. In Renaudin $B G$, the gyro bias estimation is added where, gradients update from Eq. (18) and Eq. (19) are considered. RenaudinExtaccExtmag takes both QSF detectors into account. Parameters: QSF_Window $=10, \gamma_{\mathrm{QSF} \text {-Acc }}=3.92 \mathrm{~m} \cdot \mathrm{s}^{-2}, \gamma_{\mathrm{QSF}-\mathrm{Mag}}=$ $6 \mu T$, outliersQSF_Acc $=4.90 \mathrm{~m} \cdot \mathrm{s}^{-2}$, outliersQSF_Mag $=8 \mu T$.

Sabatini et al. [30]. This filter is an EKF which considers external acceleration and magnetic perturbations as explained in Section 3. Sabatini is the common implementation of the filter. SabatiniExtacc and SabatiniExtmag takes respectively external accelerations and magnetic perturbations into account. We did not implement the gyro bias part of this filter. Parameters: $\gamma_{\text {acc }}=0.5 \mathrm{~m}_{\mathrm{s}} \mathrm{s}^{-2}, \gamma_{\mathrm{mag}}=15 \mu T, \gamma_{\theta}=10^{\circ}$, mov_average $\mathrm{mag}_{\mathrm{mag}}=0.1 \mathrm{~s}$

$\boldsymbol{E} \boldsymbol{k} \boldsymbol{f}$ is the common implementation of the Extended KF.

OS The Android API of Nexus 5 and iOS API of iPhones also provides quaternions generated by undisclosed "black-box" algorithms. We include them in our comparisons. 


\section{Experimental Protocol}

In this section, we explain our experimental methodology. A total of 126 trials have been conducted by 3 peoples with 3 different smartphones, following several typical motions in an environment with low and high magnetic disturbances.

\subsection{Ground Truth}

Reference measurements have been made by a Qualisys system. This technology provides quaternions with a precision of less than $0.5^{\circ}$ of rotation. Our room is equipped with 20 Oqus cameras connected to a server and a Qualisys Tracker software with a sampling rate at $150 \mathrm{~Hz}$. For the purpose of aligning timestamps of our ground truth data with the one of smartphone's sensors, we used a slerp interpolation [45]. The motion tracker reference frame has been aligned with $E F$ using room orientation provided by architects. The room is a $10 \mathrm{~m} \times 10 \mathrm{~m}$ square motion lak ${ }^{2}$ (see Fig. 77). In this room, we observed that the magnetic field is almost homogeneous from a sub-place to another (variations are less than $3 \mu T$ ), and with negligible variations over time.

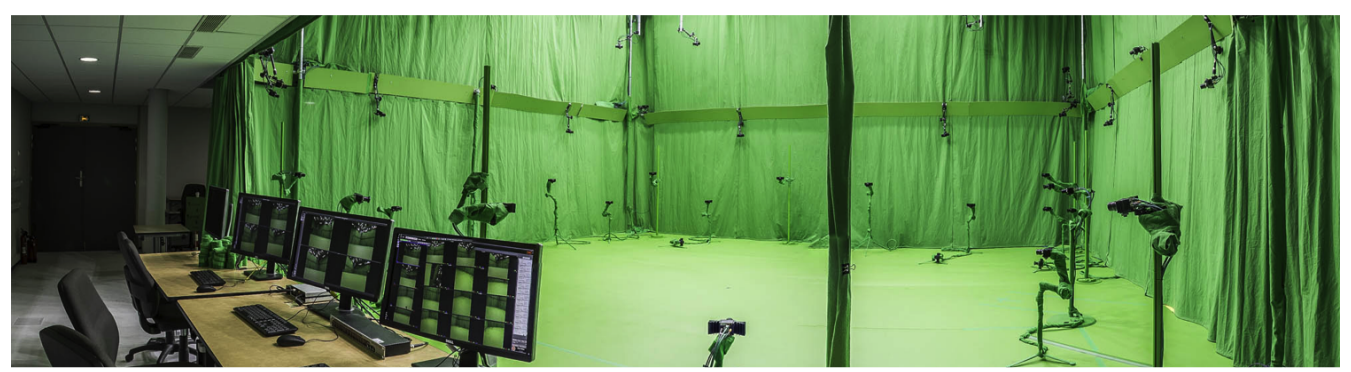

Figure 7: Kinovis room at Inria, Grenoble, France.

A smartphone handler with infrared markers has been created with a 3D printer for this study and its markers have been aligned with $S F$ (see Fig. 8).

\subsection{Typical Smartphone Motions}

We identify 8 typical smartphone motions, inspired from [46]:

- Querying the context in augmented reality (see Fig. 8a).

- Walking while user is texting a message (see Fig. 8b.

- Walking while the user is phoning (see Fig. 8c.

- Walking with a swinging hand (see Fig. 8d).

- Walking with the device in the front pocket (see Fig. 8e).

- Walking with the device in the back pocket (see Fig. 8f).

- Running with the device in the hand (see Fig. 8g).

- Running with the device in the pocket (see Fig. 8h. 


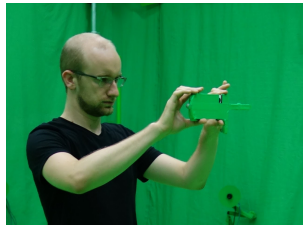

(a) AR

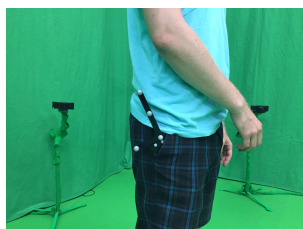

(e) Front Pocket

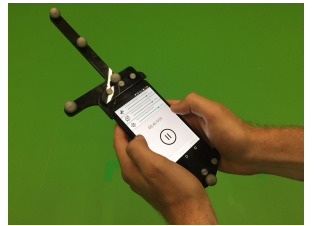

(b) Texting

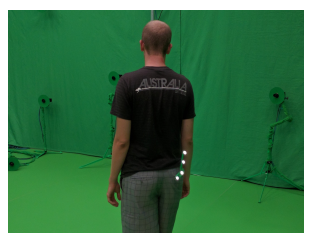

(f) Back Pocket

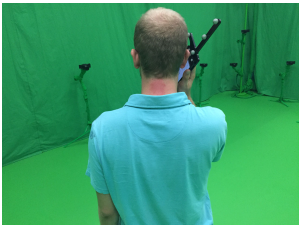

(c) Phoning

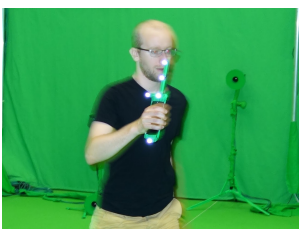

(g) Running Hand

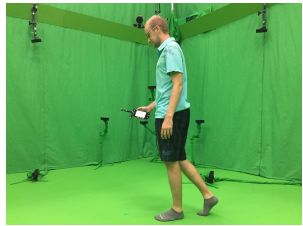

(d) Swinging

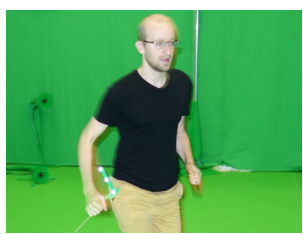

(h) Running Pocket

Figure 8: The eight typical motions for a smartphone.

AR motion is a slow motion typically found during AR experiences. Other motions happen when pedestrians move and are relevant for navigation applications. Each motion is characterized by a particular external accelerations. The Table 1 shows some statistics on external acceleration magnitude grouped by motion, for the 126 tests. The second column of Table 1 shows the average $(\mathrm{AVG})$ of external acceleration magnitude grouped by motion where the third column shows the estimated one from $\mathrm{Eq} 15$

\begin{tabular}{c|c|c|c|c|c|c|} 
& $\begin{array}{c}\text { Ext. Acc. } \\
\text { AVG } \\
\left(m . s^{-2}\right)\end{array}$ & $\begin{array}{c}\text { Ext. Acc. } \\
\text { AVG est. } \\
\left(m_{.2} s^{-2}\right.\end{array}$ & Ratio & $>0.5 m . s^{-2}$ & $>1.5 m . s^{-2}$ & $>5 m . s^{-2}$ \\
\hline AR & 0.56 & 0.24 & 2.39 & $46.4 \%$ & $2.4 \%$ & $0.0 \%$ \\
\hline Texting & 1.08 & 0.61 & 1.81 & $83.5 \%$ & $20.7 \%$ & $0.1 \%$ \\
\hline Phoning & 1.08 & 0.57 & 1.96 & $83.1 \%$ & $21.0 \%$ & $0.1 \%$ \\
\hline Front Pocket & 2.48 & 1.40 & 1.81 & $97.1 \%$ & $68.2 \%$ & $7.5 \%$ \\
\hline Back Pocket & 2.53 & 1.23 & 2.10 & $97.5 \%$ & $72.0 \%$ & $7.7 \%$ \\
\hline Swinging & 5.28 & 2.30 & 2.42 & $99.7 \%$ & $96.8 \%$ & $52.5 \%$ \\
\hline Running Pocket & 9.56 & 5.93 & 1.61 & $99.6 \%$ & $98.2 \%$ & $84.4 \%$ \\
\hline Running Hand & 16.34 & 8.44 & 2.02 & $99.9 \%$ & $99.7 \%$ & $98.6 \%$ \\
\hline
\end{tabular}

Table 1: Statistics on Magnitude of External Accelerations for each motion

During tests, we observed that external accelerations almost never reach zero because the device is always moving, and constant speed is very unlikely when the device is held or carried in a pocket. However, we noticed a high variety of external accelerations: some motions involve external accelerations that are 20 times lower than gravity while others (like running hand) are

\footnotetext{
${ }^{2}$ See: http://kinovis.inrialpes.fr
} 
closer to twice the value of gravity. We also noticed that the maximum swing of accelerometer $( \pm 2 g)$ is often reached during our running experiments.

\subsection{Different Devices}

Measurements have been recorded with 3 popular smartphones from 2 manufacturers. The 3 smartphones used are a LG Nexus 5, an iPhone 5 and an iPhone 4S. Each of them embeds, a 3-axis accelerometer, a 3-axis magnetometer and a 3-axis gyroscope. The sensors used in commodity smartphones are not directly built by the smartphone manufacturers but they are designed by third-party companies (e.g ST Micro, AKM, InvenSense). It is not uncommon that the same sensor is used for an Android and for an iOS device. The software layers (for calibration and estimation) embedded in the two operating systems (Android and iOS) are not publicly described. For this reason, we include both Android and iOS in our tests and we refer to their embedded estimation methods as black-box algorithms.

Table 2 summarizes sensors specifications for the 3 devices used in this work.

\begin{tabular}{c|c|c|c|} 
& Accelerometer & Gyroscope & Magnetometer \\
\hline \multirow{2}{*}{ iPhone 4S } & STMicro STM33DH & STIMicro AGDI & AKM 8975 \\
& $100 H z$ & $100 \mathrm{~Hz}$ & $40 \mathrm{~Hz}$ \\
\hline \multirow{2}{*}{ iPhone 5 } & STMicro LIS331DLH & STIMicro L3G4200D & AKM 8963 \\
& $100 \mathrm{~Hz}$ & $100 \mathrm{~Hz}$ & $25 \mathrm{~Hz}$ \\
\hline \multirow{2}{*}{ Nexus 5 } & InvenSense MPU6515 & InvenSense MPU6515 & AKM 8963 \\
& $200 \mathrm{~Hz}$ & $200 \mathrm{~Hz}$ & $60 \mathrm{~Hz}$ \\
\hline
\end{tabular}

Table 2: Sensors specifications with the max. sampling rate

We implemented a log application ${ }^{3}$ for Android and iOS. For the purpose of aligning timestamps of magnetic field and gyroscope data with data obtained from accelerometer, we used a linear extrapolation. In order to keep the focus on a real-time process, interpolation is not allowable here. We choose to align data at $100 \mathrm{~Hz}$. Moreover, for each trial, we chose to process 31 algorithms at 4 sampling rates and with 7 different calibrations, that is a total of more than 110 000 tests and 804 millions quaternions compared.

\subsection{Common Basis of Comparison and Reproducibility}

To ensure a reasonably fast convergence of algorithms, we initialize the first quaternion (for estimation algorithms) using the first measurement of accelerometer and the first measurement of magnetometer. In addition, we discard the first five seconds from our results, to allow time for filter to converge.

Most smartphone APIs (including Nexus 5 and iPhones) provide both calibrated and uncalibrated data from magnetometer and gyroscope ${ }^{4}$ and only uncalibrated data from accelerometer. Calibration phases can be triggered by the Android operating system at anytime. However, we notice that the gyroscope bias is removed during static phases and the magnetometer is calibrated during the drawing of an infinity symbol. For iOS devices, magnetometer calibration must be

3 https://github.com/tyrex-team/senslogs

${ }^{4}$ not from iOS API 
explicitly triggered by the user. The exact calibration algorithms embedded in both iOS and Android are not disclosed so we consider them as "black-boxes".

The precision error is reported using the Mean Absolute Error (MAE) on the Quaternion Angle Difference (QAD) [47]. It allows to avoid the use of Euler angles with the gimbal-lock problem. The formula of QAD is defined by:

$$
\theta=\cos ^{-1}\left(2\langle q 1, q 2\rangle^{2}-1\right) \text {. }
$$

Since the accuracy of the system that provides the ground truth is $\pm 0.5^{\circ}$, we consider that two algorithms exhibiting differences in precision lower than $0.5^{\circ}$ rank similarly.

\section{Comparative Analysis and Discussions}

We made available the whole benchmark including the $110000+$ of 2 -minute results and the 126 datasets at: http://tyrex.inria.fr/mobile/benchmarks-attitude. Tests can thus be reproduced. This benchmark makes it possible to evaluate new filters over a common ground truth, and to compute additional analytics like e.g. precision errors using Euler angles. In this Section we report on a few discussions, backed by aggregated views on a fraction of the obtained results.

\subsection{Importance of Calibration}

To investigate the impact of calibration, we also developed a custom calibration procedure: every morning, we applied following calibrations:

- Accelerometer - We implemented the calibration proposed by Frosio et al. in [13]. Furthermore, we applied a scale to measurements in order to adjust magnitude to $9.8 \mathrm{~m} . \mathrm{s}^{-2}$.

- Magnetometer - We implemented the calibration propose by Bartz et al. in [17] to remove soft and hard iron distortions. Moreover, we scaled the measurements to obtain the same magnitude than the Earth's magnetic field.

- Gyroscope - We simply removed the bias by subtracting measured values in each axis during static phases.

We tested attitude estimation algorithms in 6 different situations where magnetometer, gyroscope and accelerometer are either calibrated or not. These 6 situations will help us to understand the impact of each sensor calibration on the precision of attitude estimation algorithms. We present the obtained results in Table 3. The first line indicates if the sensor is calibrated by our procedure described above (Yes), if it is calibrated by the Operation System (OS) or if it is not calibrated (No).

The main observation drawn from this calibration study is that the precision is impacted in the same way with all algorithms.

In a context free from magnetic perturbations, the magnitude of uncalibrated magnetic field is about $350 \mu T$. This is why it is impossible to estimate attitude if calibration of hard iron distortions has not be done before. The gyroscope calibration phase is mostly important during periods with no update from accelerometer and magnetometer values. If gyroscope is not calibrated, integration drift will grow from $5^{\circ} \cdot \mathrm{min}^{-1}$ to $20^{\circ} \cdot \mathrm{min}^{-1}$. We observe that accelerometer calibration does not significantly improve the precision of attitude estimation for the considered datasets. The way we performed calibration provides a significantly better precision in attitude estimation than the calibration performed by device-embedded algorithms (i.e: from $18^{\circ}$ to $8^{\circ}$ for the most precise). 


\begin{tabular}{r|c|c|c|c|c|c|} 
& $\begin{array}{c}\text { Mag: No } \\
\text { Gyr: No } \\
\text { Acc: No }\end{array}$ & $\begin{array}{c}\text { Mag: Yes } \\
\text { Gyr: No } \\
\text { Acc: No }\end{array}$ & $\begin{array}{c}\text { Mag: Yes } \\
\text { Gyr: No } \\
\text { Acc: Yes }\end{array}$ & $\begin{array}{c}\text { Mag: Yes } \\
\text { Gyr: Yes } \\
\text { Acc: No }\end{array}$ & $\begin{array}{c}\text { Mag: Yes } \\
\text { Gyr: Yes } \\
\text { Acc: Yes }\end{array}$ & $\begin{array}{c}\text { Mag: OS } \\
\text { Gyr: OS* } \\
\text { Acc: No }\end{array}$ \\
\hline Choukroun & $95.1^{\circ}$ & $16.5^{\circ}$ & $16.5^{\circ}$ & $9.9^{\circ}$ & $\mathbf{1 0 . 0}^{\circ}$ & $17.3^{\circ}$ \\
\hline Fourati & $83.7^{\circ}$ & $15.6^{\circ}$ & $15.5^{\circ}$ & $10.3^{\circ}$ & $\mathbf{1 0 . 4}^{\circ}$ & $16.3^{\circ}$ \\
\hline Madgwick & $77.5^{\circ}$ & $18.2^{\circ}$ & $18.2^{\circ}$ & $8.1^{\circ}$ & $\mathbf{8 . 1}^{\circ}$ & $17.7^{\circ}$ \\
\hline Mahony & $99.6^{\circ}$ & $20.2^{\circ}$ & $20.2^{\circ}$ & $14.2^{\circ}$ & $\mathbf{1 4 . 2}^{\circ}$ & $19.1^{\circ}$ \\
\hline Renaudin & $82.2^{\circ}$ & $19.5^{\circ}$ & $19.5^{\circ}$ & $8.0^{\circ}$ & $\mathbf{8 . 1}^{\circ}$ & $18.1^{\circ}$ \\
\hline Ekf & $79.8^{\circ}$ & $19.4^{\circ}$ & $19.4^{\circ}$ & $7.9^{\circ}$ & $\mathbf{8 . 0}^{\circ}$ & $18.2^{\circ}$ \\
\hline
\end{tabular}

${ }^{*}$ Not available for iOS devices

Table 3: Precision of attitude estimation according to calibration with all motions

\subsection{The Difficulty with Noise for Kalman Filters}

Kalman Filters (KFs) are often used in the general domain of attitude estimation where white noises naturally model physical sensors noise. We know from theory that KF converge when the smartphone is static and magnetometer values correspond to Earth's magnetic field. However, this is not the case in the context that we consider. The magnitude of external accelerations and magnetic perturbations experienced by the smartphone is much higher than its physical sensors noise.

With values for sensors noise experimentally extracted (as commonly found in the literature), filters yield high precision errors and diverge quickly. This is shown in Table 4 where ChoukrounSn, RenaudinSn and EkfSn respectively denote the algorithms initialized with values for noise measured from physical sensors.

\begin{tabular}{|c|c|c|c|c|c|c|c|c|}
\hline & 产 & 怘 & 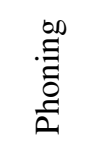 & 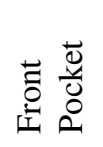 & 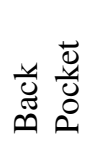 & 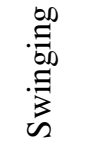 & 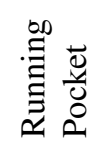 & 䓃 \\
\hline Choukroun & $5.1^{\circ}$ & $4.3^{\circ}$ & $4.4^{\circ}$ & $4.8^{\circ}$ & $4.6^{\circ}$ & $6.3^{\circ}$ & $7.9^{\circ}$ & $21.1^{\circ}$ \\
\hline ChoukrounSn & $15.6^{\circ}$ & $20.6^{\circ}$ & $15.9^{\circ}$ & $17.8^{\circ}$ & $16.9^{\circ}$ & $11.5^{\circ}$ & $17.6^{\circ}$ & $35.2^{\circ}$ \\
\hline$E k f$ & $4.5^{\circ}$ & $4.0^{\circ}$ & $3.7^{\circ}$ & $4.6^{\circ}$ & $4.6^{\circ}$ & $5.9^{\circ}$ & $8.2^{\circ}$ & $16.8^{\circ}$ \\
\hline$E k f S n$ & $44.0^{\circ}$ & $57.8^{\circ}$ & $36.1^{\circ}$ & $20.6^{\circ}$ & $30.8^{\circ}$ & $29.1^{\circ}$ & $23.3^{\circ}$ & $54.1^{\circ}$ \\
\hline Renaudin & $4.5^{\circ}$ & $3.8^{\circ}$ & $3.7^{\circ}$ & $4.7^{\circ}$ & $4.6^{\circ}$ & $6.1^{\circ}$ & $8.5^{\circ}$ & $17.9^{\circ}$ \\
\hline RenaudinSn & $20.8^{\circ}$ & $18.5^{\circ}$ & $17.8^{\circ}$ & $17.3^{\circ}$ & $18.4^{\circ}$ & $11.4^{\circ}$ & $17.4^{\circ}$ & $36.5^{\circ}$ \\
\hline
\end{tabular}

Table 4: Precision of attitude estimation according to sensor noises without magnetic perturbations.

KFs can still give better results in this context, provided we adapt the "noise values" in a way that does not reflect anymore physical sensors noise, but that instead takes into account the relative importance of sensor measurements in this context. Gyroscope measurements are not impacted by external accelerations nor magnetic perturbations. In our context, we observed that giving more importance to gyroscope measurements (compared to magnetometer and accelerometer measurements) yields better results (despite convergence being a bit longer). Experimentally 
we obtained the best results (See Choukroun, Renaudin and $E k f$ in Table 4) by using the following "noise values": $\sigma_{\mathrm{acc}}=0.5, \sigma_{\mathrm{mag}}=0.8, \sigma_{\mathrm{gyr}}=0.3$ for all $\mathrm{KF} 5$.

Applying KFs remains non trivial, because the notion of noise to model in this context goes much beyond the setting in which initial KFs were designed.

Observers and KFs exhibit similar results for low to moderate external accelerations. For higher accelerations (typically found when swinging and running), observers were found to improve precision. This is especially the case for Fourati that outperforms $E k f$, as shown in Table 6

\subsection{Bias Consideration}

Many existing filters try to estimate sensors bias and in particular gyroscope bias. For example, in observers, typical procedures use residuals between reference and estimation to estimate bias (e.g. [37, 39]). In our setting however, residuals do not only originate from gyroscope bias but also from magnetic perturbations and external accelerations. Furthermore, a calibration phase is performed in a previous stage.

We can thus wonder how useful classical bias estimation techniques are in our setting. Table 5 compares the results obtained with two variants of each filter: one with bias estimation and one without. We observe that bias estimation seems unnecessary in our context of study. We remark however that bias estimation can still be useful for situations where the gyroscope is not calibrated. In this particular case, precision of attitude estimation is improved with bias estimation, provided external accelerations remain small.

\begin{tabular}{|c|c|c|c|c|c|c|c|c|}
\hline & $\frac{2}{4}$ & . & 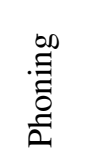 & 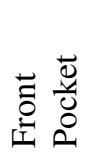 & 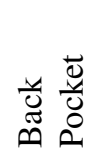 & 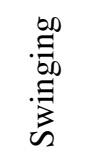 & 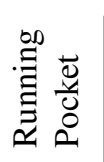 & 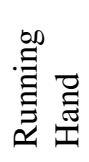 \\
\hline Madgwick & $4.8^{\circ}$ & $4.1^{\circ}$ & $4.6^{\circ}$ & $4.9^{\circ}$ & $5.0^{\circ}$ & $5.8^{\circ}$ & $7.1^{\circ}$ & $16.5^{\circ}$ \\
\hline MadgwickB & $5.2^{\circ}$ & $4.8^{\circ}$ & $5.4^{\circ}$ & $5.8^{\circ}$ & $6.2^{\circ}$ & $11.5^{\circ}$ & $10.5^{\circ}$ & $19.8^{\circ}$ \\
\hline Mahony & $5.0^{\circ}$ & $4.6^{\circ}$ & $4.2^{\circ}$ & $5.1^{\circ}$ & $5.2^{\circ}$ & $7.5^{\circ}$ & $7.9^{\circ}$ & $11.2^{\circ}$ \\
\hline MahonyB & $5.6^{\circ}$ & $4.9^{\circ}$ & $4.7^{\circ}$ & $6.1^{\circ}$ & $5.7^{\circ}$ & $9.9^{\circ}$ & $13.1^{\circ}$ & $26.4^{\circ}$ \\
\hline Renaudin & $4.5^{\circ}$ & $3.8^{\circ}$ & $3.7^{\circ}$ & $4.7^{\circ}$ & $4.6^{\circ}$ & $6.1^{\circ}$ & $8.5^{\circ}$ & $17.9^{\circ}$ \\
\hline RenaudinBG & $4.5^{\circ}$ & $3.7^{\circ}$ & $3.8^{\circ}$ & $4.5^{\circ}$ & $4.6^{\circ}$ & $6.9^{\circ}$ & $12.8^{\circ}$ & $19.3^{\circ}$ \\
\hline
\end{tabular}

Table 5: Precision of attitude according to bias estimation without magnetic perturbations.

\subsection{Behaviors during Typical Smartphone Motions}

Table 6 compares the precision of attitude estimation for each motion without magnetic perturbations. We observe a negative correlation between magnitude of external accelerations (Avg of Ext. Acc.) and precision of attitude estimation. This is verified for all algorithms.

First, we observe that the precisions obtained with all algorithms are roughly similar whenever external accelerations are low (from AR to Back Pocket). However, we observe that one algorithm stands out in terms of precision: Fourati in the case of high external accelerations (Swinging and Running).

\footnotetext{
${ }^{5}$ except for the Linear KF from Choukroun where we adapt these values for the linearized model: $\sigma_{\mathrm{acc}}=0.3, \sigma_{\mathrm{mag}}=$ $0.3, \sigma_{\mathrm{gyr}}=0.5$
} 


\begin{tabular}{|c|c|c|c|c|c|c|c|c|}
\hline & 学 & 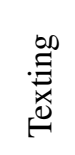 & $\begin{array}{l}\stackrel{\infty}{D} \\
\stackrel{\Xi}{0} \\
\frac{0}{2}\end{array}$ & 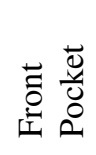 & 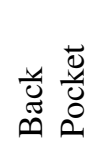 & 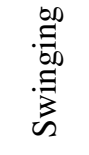 & 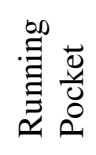 & 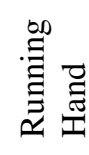 \\
\hline Avg of Ext. Acc. $\left(m . s^{-2}\right)$ & 0.56 & 1.08 & 1.08 & 2.48 & 2.53 & 5.28 & 9.56 & 16.34 \\
\hline black-box & $7.1^{\circ}$ & $5.9^{\circ}$ & $5.8^{\circ}$ & $12.7^{\circ}$ & $13.2^{\circ}$ & $20.3^{\circ}$ & $24.4^{\circ}$ & $62.0^{\circ}$ \\
\hline Choukroun & $5.1^{\circ}$ & $4.3^{\circ}$ & $4.4^{\circ}$ & $4.8^{\circ}$ & $4.6^{\circ}$ & $6.3^{\circ}$ & $7.9^{\circ}$ & $21.1^{\circ}$ \\
\hline Madgwick & $4.8^{\circ}$ & $4.1^{\circ}$ & $4.6^{\circ}$ & $4.9^{\circ}$ & $5.0^{\circ}$ & $5.8^{\circ}$ & $7.1^{\circ}$ & $16.5^{\circ}$ \\
\hline Mahony & $5.0^{\circ}$ & $4.6^{\circ}$ & $4.2^{\circ}$ & $5.1^{\circ}$ & $5.2^{\circ}$ & $7.5^{\circ}$ & $7.9^{\circ}$ & $11.2^{\circ}$ \\
\hline Fourati & $4.8^{\circ}$ & $4.0^{\circ}$ & $4.4^{\circ}$ & $4.6^{\circ}$ & $4.8^{\circ}$ & $5.3^{\circ}$ & $6.3^{\circ}$ & $6.6^{\circ}$ \\
\hline FouratiExtacc & $4.9^{\circ}$ & $5.4^{\circ}$ & $4.7^{\circ}$ & $6.0^{\circ}$ & $5.7^{\circ}$ & $8.4^{\circ}$ & $12.2^{\circ}$ & $29.1^{\circ}$ \\
\hline Sabatini & $4.5^{\circ}$ & $4.0^{\circ}$ & $3.7^{\circ}$ & $4.6^{\circ}$ & $4.6^{\circ}$ & $5.9^{\circ}$ & $8.2^{\circ}$ & $16.8^{\circ}$ \\
\hline SabatiniExtacc & $4.5^{\circ}$ & $4.5^{\circ}$ & $4.0^{\circ}$ & $5.5^{\circ}$ & $5.0^{\circ}$ & $9.7^{\circ}$ & $15.0^{\circ}$ & $33.5^{\circ}$ \\
\hline Renaudin & $4.5^{\circ}$ & $3.8^{\circ}$ & $3.7^{\circ}$ & $4.7^{\circ}$ & $4.6^{\circ}$ & $6.1^{\circ}$ & $8.5^{\circ}$ & $17.9^{\circ}$ \\
\hline RenaudinExtacc & $4.5^{\circ}$ & $3.8^{\circ}$ & $3.7^{\circ}$ & $4.8^{\circ}$ & $4.8^{\circ}$ & $6.0^{\circ}$ & $8.0^{\circ}$ & $30.3^{\circ}$ \\
\hline
\end{tabular}

Table 6: Precision of attitude estimation according to typical motions without magnetic perturbations.

We also observe that filters which take external accelerations into account do not yield better precision than others. In Table 1], we present the left term $\mu$ of detector (Eq. (15p) and the magnitude of external accelerations (extracted from the ground truth). We observe that the two series are highly correlated $(\rho>99 \%)$. This suggests that it is possible to reasonably distinguish periods with high external accelerations, but, in practice this is not verified. This can be explained by long periods of perturbations without the smartphone becoming completely static (see the last 3 columns of Table 1 for high motions [Swinging and Running]). Moreover, filters are very sensitive to false detections which make them quickly diverge. For example, if the user puts a force of $-2 g$ on the device in direction of the sky, the magnitude of measured acceleration will be $g$ and unfortunately the detector will not reject this high external acceleration. Conversely, if a force in the same direction than the gravity with a magnitude greater than $\gamma_{a c c}$ is applied on the device, the measurement will be rejected by the detector whereas attitude will be well estimated. An interesting perspective for the further development of filters in this context would be to investigate how to better leverage the detection of periods with high external accelerations in order to improve precision of attitude estimation during those periods (Table 6.

\subsection{Comparison with Device-Embedded Algorithms}

Table 7 shows algorithms precision depending on the smartphone used.

For each algorithm (except for the black-box ones), we observe similar results across the iPhone 5 and the Nexus 5. Results with iPhone $4 \mathrm{~S}$ are slightly more precise than the ones obtained with the two other devices. Contrary to what one might think, the recent smartphones do not exhibit a better behavior than the older ones. This difference can be explained by the quality of sensors used (see Section 5.3).

We also observe that all algorithms exhibit a similar or better precision compared to OSembedded algorithms. We know that this is at least partially due to a bad calibration (especially for iPhones). 


\begin{tabular}{r|c|c|c|} 
& iPhone 4S & iPhone 5 & LG Nexus 5 \\
\hline black-box & $23.6^{\circ}$ & $28.6^{\circ}$ & $12.7^{\circ}$ \\
\hline Choukroun & $8.6^{\circ}$ & $10.4^{\circ}$ & $10.9^{\circ}$ \\
\hline Madgwick & $7.1^{\circ}$ & $8.7^{\circ}$ & $8.6^{\circ}$ \\
\hline Mahony & $10.8^{\circ}$ & $15.2^{\circ}$ & $16.6^{\circ}$ \\
\hline Fourati & $8.8^{\circ}$ & $10.3^{\circ}$ & $12.1^{\circ}$ \\
\hline Ekf & $6.7^{\circ}$ & $8.7^{\circ}$ & $8.5^{\circ}$ \\
\hline
\end{tabular}

Table 7: Precision according to device with all motions and with/without magnetic perturbations.

On 126 tests, we noticed that the Ekf improves the precision of OS-embedded algorithms on iPhone $4 \mathrm{~S}$ by $250 \%$, iPhone 5 by $230 \%$ and Nexus 5 by $50 \%$.

\subsection{Empirical Computational Complexity}

Because of smartphone's limited resources (e.g. battery), we study to which extent improvements in precision of attitude estimation have an impact in terms of empirical computational complexity. Figure 9 summarizes the relative times spent with each algorithm, where unit time corresponds to the running time of Mahony. Ratios have been obtained using the offline implementations executed across all 126 datasets.

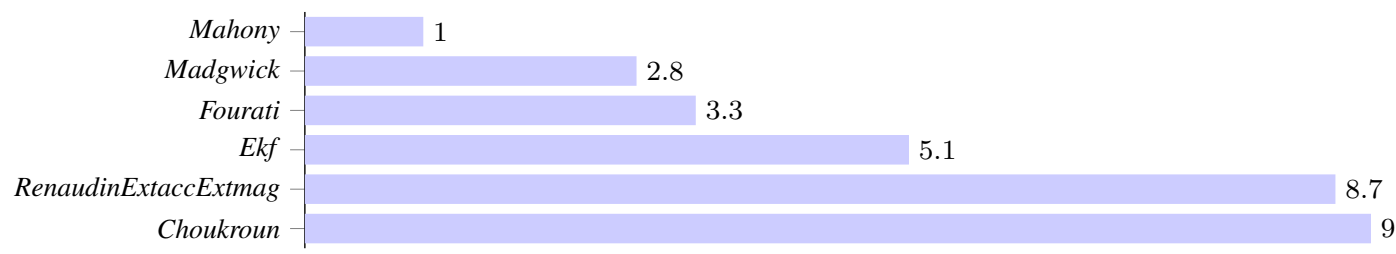

Figure 9: Relative performance in terms of CPU cost (lower is better).

We observe that all algorithms can be executed on smartphones even at much higher frequencies than current sensors capabilities (see Table 2). For example, our implementation of Mahony running on the Nexus 5 can output up to 45000 quaternions per second, that is to say, it consumes up to $1.5 \%$ of the smartphone's CPU at $200 \mathrm{~Hz}$.

\subsection{Relevant Sampling Rates}

In all aforementioned results, sensors sampling rate was set to $100 \mathrm{~Hz}$. We studied the behavior of algorithms whenever the sampling rate varies. Table 8 presents precision according to sampling rate.

We observe that results with a sampling at $100 \mathrm{~Hz}$ and $40 \mathrm{~Hz}$ are relatively similar, and much more precise than with lower frequencies. This suggests to implement filters with a sampling rate of $40 \mathrm{~Hz}$ to save smartphone's battery life, for a negligible loss in precision.

In our specific context of a smartphone held by a pedestrian, we obtain a mean error of $8^{\circ}$ using the best algorithm $(E k f)$. This might be suitable for a navigation application with short trips. For longer trips, the additional use of a map-matching algorithm might be considered. 
Table 8: Precision according to sampling with all motions and with/without magnetic perturbations.

\begin{tabular}{r|c|c|c|c|} 
& $100 \mathrm{~Hz}$ & $40 \mathrm{~Hz}$ & $10 \mathrm{~Hz}$ & $2 \mathrm{~Hz}$ \\
\hline Choukroun & $10.0^{\circ}$ & $10.1^{\circ}$ & $15.6^{\circ}$ & $34.7^{\circ}$ \\
\hline Mahony & $14.2^{\circ}$ & $14.3^{\circ}$ & $19.7^{\circ}$ & $48.9^{\circ}$ \\
\hline Madgwick & $8.1^{\circ}$ & $8.1^{\circ}$ & $17.3^{\circ}$ & $62.8^{\circ}$ \\
\hline Fourati & $10.4^{\circ}$ & $10.4^{\circ}$ & $18.9^{\circ}$ & $52.5^{\circ}$ \\
\hline Ekf & $8.0^{\circ}$ & $8.1^{\circ}$ & $15.3^{\circ}$ & $49.5^{\circ}$ \\
\hline
\end{tabular}

\section{Limiting the Impact of Magnetic Perturbations}

The presence of magnetic perturbations in indoor environments is well-known [44]. For example, Figure 10 illustrates variations of the magnetic field magnitude we observed inside Inria's research center (Grenoble, France) compared to Earth's magnetic field. Our measurements correspond to the total field perturbation observed in [15]. However, the study from Afzal et al. does not remark that high perturbations are more prone to occur when a smartphone is held close to particular objects found in buildings. For instance, we notice that when the smartphone is held at less than $50 \mathrm{~cm}$ away from a ferro-magnetic heater or less than $1 \mathrm{~m}$ away from an electrical cabinet, the magnitude of the magnetic field can grow up to $150 \mu T$ (see Figure 10), which is 3 times greater than Earth's magnetic field.

\subsection{A New Algorithm for Better Limitation of Magnetic Perturbations Impact}

To limit the impact of such magnetic perturbations, we propose a new approach that further builds on the idea of detectors à la (16). The overall principle is twofold: (1) during periods when we detect magnetic perturbations, we can discard magnetometer measurements for a short period $(\approx 2-3 s)$ so that more importance are given to gyroscope measurements; $(2)$ this period should be reasonably short-enough so that the impact of gyroscope's bias ${ }^{6}$ is limited.

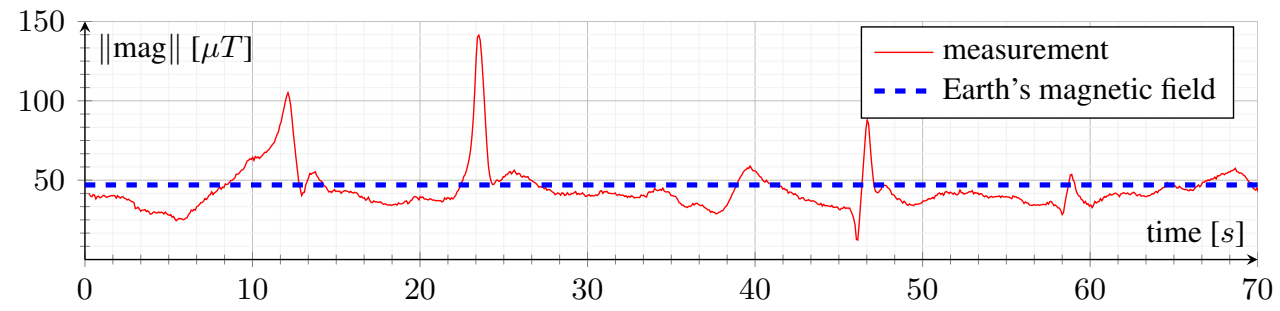

Figure 10: Magnitude of magnetic field measurements and Earth's magnetic field in the indoor environment of Inria building in Grenoble.

We propose an improvement of the magnetic perturbation detector (Eq. (16) ) adapted to the pedestrian context. When a person is moving with a normal speed (walk) in a building, we have observed huge variations of magnitude of magnetic field $\|{ }^{S}$ mag $\|>100 \mu T$ (see for example Fig. 10 at $t=24 s$ ). The main problem with the detector $(16)$ is to find a proper

\footnotetext{
${ }^{6}$ We experimentally measured the drift due to gyroscope's bias integration as approximately $5{ }^{\circ} / \mathrm{min}$.
} 
$\gamma_{\text {mag }}$ which should be: (i) high enough not to discard magnetometer measurements due to low magnetic perturbations omnipresent in an indoor environment and (ii) small enough to reject high perturbations which affect attitude estimation (such as those coming from the proximity of e.g. heaters, see: Section 7.2

When the threshold of (Eq. (16) is reached, generally the filter is already diverging. This means that when this detection occurs, and therefore when gyroscope integration starts, magnetometer measurements involving perturbations below the threshold have already impacted attitude estimation.

Figure 11 presents our new technique to limit the impact of magnetic perturbations. The

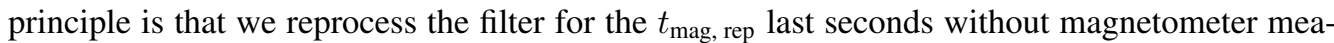
surements (Eq. (12)). When the detection occurs, attitude estimation is immediately replaced by these values. This technique avoids the attitude divergence during the $t_{\text {mag, rep last seconds before }}$ the detection (Eq. (16)). This technique can be used for real-time attitude estimation (time for reprocessing being negligible when compared to $t_{\text {mag, rep }}$ ), in which case a discontinuity of some degrees can be observed when the detection occurs (see Fig. 15).

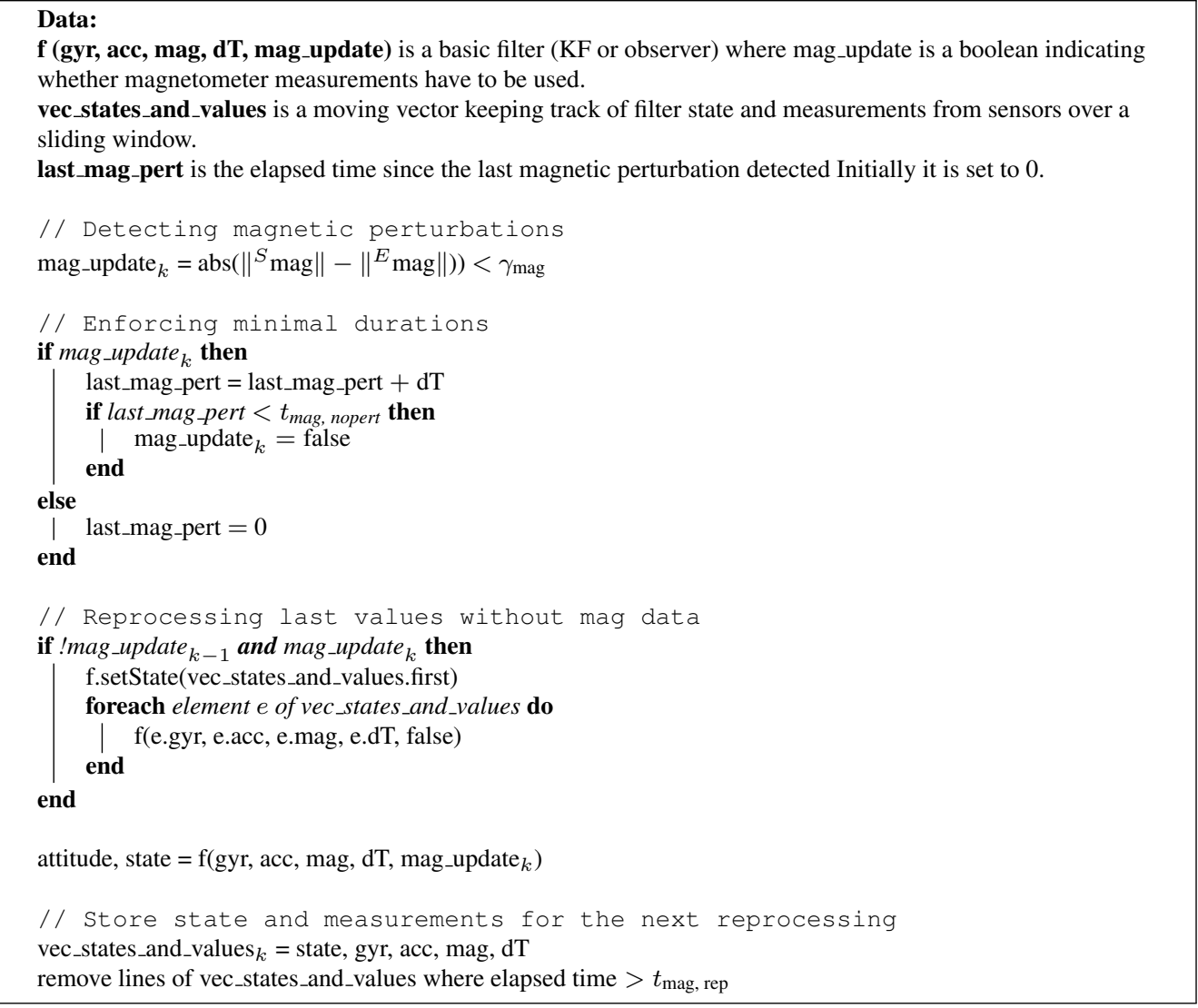

Figure 11: Pseudo-code for limiting the impact of magnetic perturbations.

During periods of magnetic perturbation, Eq. 16 can be true for a small duration. This 
is because magnitude of magnetometer measurement can be similar to Earth's magnetic field magnitude during a perturbation phase, it depends on the direction of the perturbation. For this purpose a last condition is added: Eq. (12) can be used only if there is no detection (Eq. (16) during the last $t_{\mathrm{mag}, \text { nopert }}$ seconds.

This technique works with all filters where updates (Eq. (11)) from magnetometer can be temporarily removed (which is the case of all algorithms considered here). An important prerequisite is magnetometer calibration. In a context without magnetic perturbations, magnitude of magnetometer measurements should be equal to the magnitude of Earth's magnetic field.

In addition to the algorithms presented previously in Section 4 , we also consider 2 new algorithms based on the aforementioned technique. The first one, MichelObsF, is an implementation of the technique where $f(\mathrm{gyr}, \mathrm{acc}$, mag, dT, mag_update) is the observer function from Fourati et al. [12] modified to take into account the new parameter mag_update. The whole algorithm is given below.

Algorithm - MichelObsF

Complementary Filter Algorithm

$$
\begin{aligned}
{ }_{\mathrm{acc}_{q, t}} & =\hat{q}_{t-1}^{-1} \otimes{ }^{E} \operatorname{acc}_{q, t} \otimes \hat{q}_{t-1} \\
{ }^{S} \operatorname{mâg}_{q, t} & =\hat{q}_{t-1}^{-1} \otimes{ }^{E} \operatorname{mag}_{q, t} \otimes \hat{q}_{t-1}
\end{aligned}
$$

if (mag_update)

$$
X=2\left[K_{a}\left[{ }^{S} \mathrm{acc}_{t} \times\right] \quad K_{m}\left[{ }^{S} \operatorname{mâg}_{t}{ }^{\times}\right]\right]^{T}
$$

else

$$
X=2\left[\begin{array}{ll}
K_{a}\left[{ }^{S} \hat{a c c}_{t} \times\right. & 0_{3 \times 3}
\end{array}\right]^{T}
$$

end

$$
\begin{aligned}
& K=\left[X^{T} X+\lambda I_{3 \times 3}\right]^{-1} X^{T} \text {, with } \lambda=10^{-5} \\
& \hat{q}_{e, t}=\left[K\left[\begin{array}{c}
1 \\
{ }^{S} \operatorname{acc}_{t}-{ }^{S} \mathrm{accc}_{t} \\
{ } \operatorname{mag}_{t}-{ }^{S} \text { mâg }_{t}
\end{array}\right]\right] \\
& \dot{\hat{q}}_{t}=\frac{1}{2} \hat{q}_{t-1} \otimes{ }^{S} \mathrm{gyr}_{q, t} \otimes \beta \hat{q}_{e, t} \\
& \hat{q}_{t}=\hat{q}_{t-1}+\dot{\hat{q}}_{t} * \Delta t
\end{aligned}
$$

where, $v^{\times}$is the skew matrix of 3D vector $v$.

$$
\left[v^{\times}\right]=\left[\begin{array}{ccc}
0 & -v_{z} & v_{y} \\
v_{z} & 0 & -v_{x} \\
-v_{y} & v_{x} & 0
\end{array}\right]
$$


The second algorithm, MichelEkfF, is designed such that $\mathrm{f}$ corresponds to the well known EKF filter from the literature [10]. The whole algorithm is described below.

\section{Algorithm - MichelEkfF}

\section{Extended Kalman Filter}

State Vector:

$$
q=\left[\begin{array}{llll}
q_{w} & q_{x} & q_{y} & q_{z}
\end{array}\right]
$$

Prediction:

$$
\begin{aligned}
\Omega_{t} & =\frac{1}{2}\left[\begin{array}{cc}
0 & -{ }^{S} \mathrm{gyr}_{t} \\
{ } \mathrm{gyr}_{t}^{T} & -\left[{ }^{S} \operatorname{gyr}_{t} \times\right]
\end{array}\right] \Delta t \\
\hat{q}_{t} & =\exp \left(\Omega_{t}\right) \times q_{t-1}
\end{aligned}
$$

\section{Correction:}

$$
\begin{aligned}
& \delta z_{t}=\left[\begin{array}{c}
{ }^{E} \operatorname{mag}_{q, t}-\hat{q}_{t} \otimes{ }^{S} \operatorname{mag}_{q, t} \otimes \hat{q}_{t}^{-1} \\
{ }^{E} \operatorname{acc}_{q, t}-\hat{q}_{t} \otimes{ }^{S} \operatorname{acc}_{q, t} \otimes \hat{q}_{t}^{-1}
\end{array}\right] \\
& H_{t}=\left[\begin{array}{c}
\text { jacobian }\left(\hat{q}_{t} \otimes{ }^{S} \operatorname{mag}_{q, t} \otimes \hat{q}_{t}^{-1}\right) \\
j \operatorname{acobian}\left(\hat{q}_{t} \otimes{ }^{S} \operatorname{acc}_{q, t} \otimes \hat{q}_{t}^{-1}\right)
\end{array}\right] \\
& \text { if (mag_update) } \\
& R=\left[\begin{array}{ll}
\sigma_{\text {mag }} & 0_{3 \times 3} \\
0_{3 \times 3} & \sigma_{\text {acc }}
\end{array}\right]
\end{aligned}
$$

else

$$
\begin{gathered}
R=\left[\begin{array}{cc}
\lambda * I_{3 \times 3} & 0_{3 \times 3} \\
0_{3 \times 3} & \sigma_{\mathrm{acc}}
\end{array}\right] \\
\text { where: } \lambda \gg \sigma_{\mathrm{mag}}
\end{gathered}
$$

end

$$
\begin{aligned}
K_{t} & =P_{t} H_{t}^{T}\left(H_{t} P_{t} H_{t}^{T}+R\right)^{-1} \\
\hat{q}_{t} & =\hat{q}_{t}-K_{t} \delta z_{t} \\
P_{t} & =\left(I_{4 \times 4}-K_{t} H_{t}\right) P_{t}
\end{aligned}
$$

From the trials we conducted, we extracted for both algorithms the following common parameters: $\gamma_{\mathrm{mag}}=15 \mu T, t_{\mathrm{mag}, \text { nopert }}=2 \mathrm{~s}$ and $t_{\mathrm{mag} \text {, rep }}=3 \mathrm{~s}$. 


\subsection{Experimental Protocol: Introducing Magnetic Perturbations}

During tests, we noticed that magnetic disturbances are always present in indoor-environments, and they vary between different buildings. This is mainly due to the building structure. We also observed in some cases, if user is close to heaters, electrical cabinets or simply close to a wall, magnitude of magnetic field can grow up to $150 \mu T$ during few seconds, that is to say, 3 times more than Earth's magnetic field (see Fig. 10 .

The motion capture system we used is located in a room with low and constant magnetic perturbations (see Fig. 12).

In order to reproduce typical magnetic perturbations of indoor environments inside the motion lab, we used several magnetic boards (see Fig. 13). This allowed us to introduce magnetic perturbations similar to the ones described above in Fig. 10. Specifically, during the 2 minutes tests, we brought the device to a few centimeters away from magnetic boards; and we repeated this 3 or 4 times (see Fig. 14).

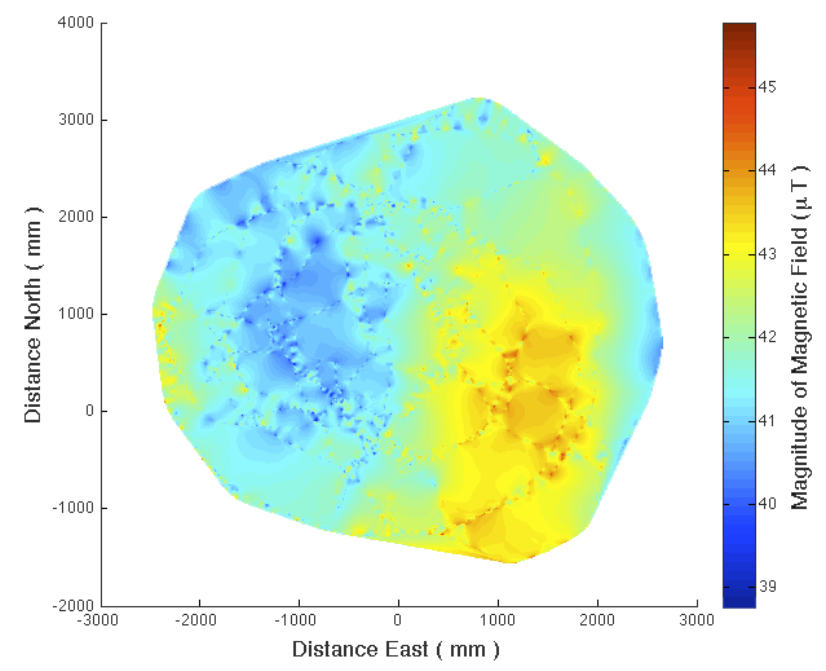

Figure 12: Heatmap of magnetic field magnitude of the motion lab.

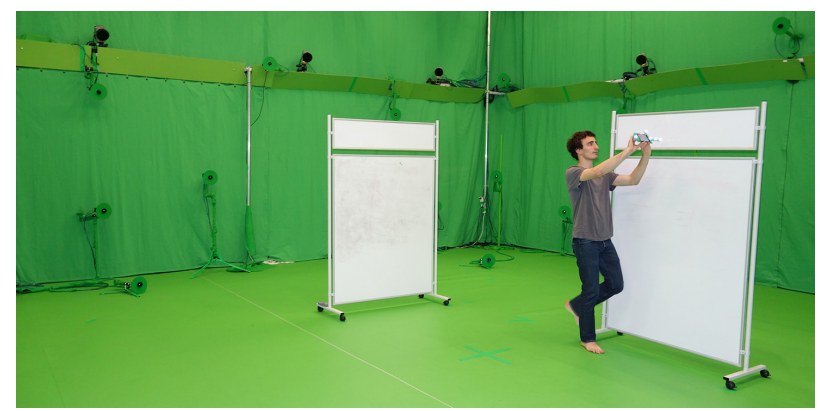

Figure 13: Magnetic boards for building structure and heaters simulation. 


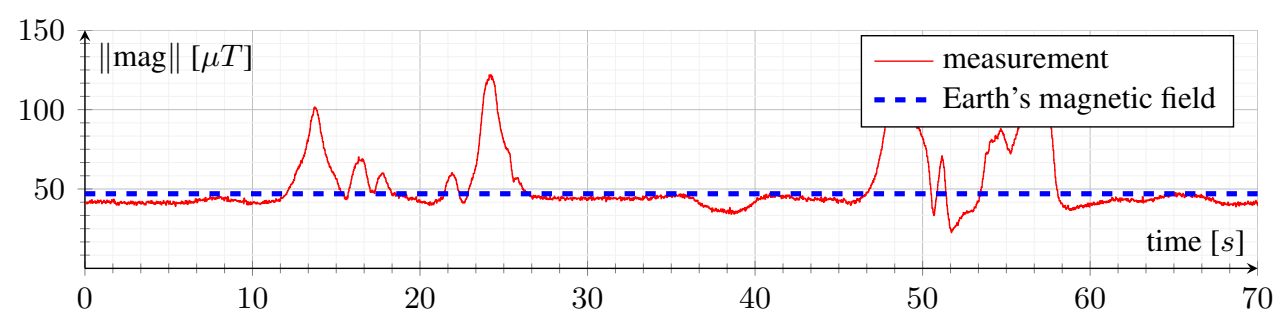

Figure 14: Magnitude of magnetic field measurements and Earth's magnetic field during our simulation with magnetic boards.

The Table 9 shows some statistics on External Magnetic Field Magnitude (EMFM). When we do not consider white magnetic boards, magnitude of magnetic field is not totally equal to the magnitude of Earth's magnetic field, so perturbations cannot be entirely omitted. If we add magnetic boards, a difference between the two magnitudes can be clearly observed (column 2). In average, $26.5 \%$ of the time, magnetic perturbations have a magnitude higher than $>5 \mu T$ and they not exist if we remove magnetic boards.

Table 9: Statistics on Magnitude of Magnetic Field with low and high magnetic perturbations

\begin{tabular}{c|c|c|c|c|c|c|c|} 
& $\begin{array}{c}\text { EMFM } \\
(\mu T)\end{array}$ & $\begin{array}{c}\text { EMFM Estimated } \\
(\mu T)\end{array}$ & Ratio & $\begin{array}{c}\text { STD } \\
(\mu T)\end{array}$ & $>0.5 \mu T$ & $>1.5 \mu T$ & $>5 \mu T$ \\
\hline High & 29.57 & 18.61 & 1.65 & 43.09 & $46.7 \%$ & $31.2 \%$ & $26.5 \%$ \\
\hline Low & 7.12 & 5.18 & 1.40 & 1.99 & $13.0 \%$ & $0.2 \%$ & $0.0 \%$ \\
\hline
\end{tabular}

\subsection{Results and Discussions}

We tested the 8 typical motions in the presence of magnetic perturbations and we showed results in Table 10 .

We observe that filters which implement a magnetic perturbations detector do not systematically exhibit a better behavior when compared to their native variant. However, if we extend them with our technique for enforcing minimal durations (See Fig. 11, precision is systematically improved when compared to their native variant. In order to observe the improvement at each step (detection, waiting-time and reprocess) of our algorithm we created intermediate filters as following:

- MichelObs and MichelEkf are common implementations of the Fourati and Ekf filters.

- In MichelObsExtmag and MichelEkfExtmag, we added magnetic perturbations detector.

- In MichelObsExtmagWt and MichelEkfExtmagWt, we added minimum duration checking.

- Finally, in MichelObsF and MichelEkfF, we added the reprocess phase. Both correspond to the whole algorithm presented in Fig. 11

RenaudinExtmag implements a different detector for magnetic perturbations based on variances which improves Renaudin. However, RenaudinExtmag is very sensitive to false detections because Earth's magnetic field is known only during the initial phase. 


\begin{tabular}{|c|c|c|c|c|c|c|}
\hline & 学 & 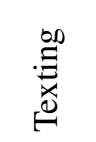 & 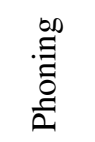 & 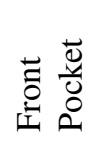 & 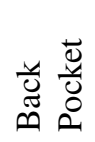 & 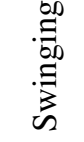 \\
\hline black-box & $29.0^{\circ}$ & $24.4^{\circ}$ & $21.1^{\circ}$ & $19.8^{\circ}$ & $37.9^{\circ}$ & $19.2^{\circ}$ \\
\hline Madgwick & $18.2^{\circ}$ & $7.5^{\circ}$ & $7.8^{\circ}$ & $8.1^{\circ}$ & $9.4^{\circ}$ & $10.0^{\circ}$ \\
\hline Mahony & $31.8^{\circ}$ & $26.1^{\circ}$ & $30.0^{\circ}$ & $19.9^{\circ}$ & $13.9^{\circ}$ & $26.6^{\circ}$ \\
\hline Renaudin & $17.1^{\circ}$ & $7.0^{\circ}$ & $7.6^{\circ}$ & $8.9^{\circ}$ & $8.7^{\circ}$ & $9.5^{\circ}$ \\
\hline RenaudinExtmag & $16.8^{\circ}$ & $6.4^{\circ}$ & $7.3^{\circ}$ & $8.4^{\circ}$ & $8.4^{\circ}$ & $8.9^{\circ}$ \\
\hline Sabatini & $16.6^{\circ}$ & $7.0^{\circ}$ & $8.0^{\circ}$ & $8.9^{\circ}$ & $8.6^{\circ}$ & $10.1^{\circ}$ \\
\hline SabatiniExtmag & $14.6^{\circ}$ & $8.7^{\circ}$ & $8.9^{\circ}$ & $6.4^{\circ}$ & $8.4^{\circ}$ & $9.0^{\circ}$ \\
\hline MichelObs & $32.1^{\circ}$ & $14.0^{\circ}$ & $16.4^{\circ}$ & $14.6^{\circ}$ & $8.8^{\circ}$ & $19.1^{\circ}$ \\
\hline MichelObsExtmag & $18.0^{\circ}$ & $11.9^{\circ}$ & $11.4^{\circ}$ & $7.4^{\circ}$ & $8.8^{\circ}$ & $10.3^{\circ}$ \\
\hline MichelObsExtmagWt & $15.5^{\circ}$ & $9.2^{\circ}$ & $9.7^{\circ}$ & $7.1^{\circ}$ & $7.3^{\circ}$ & $10.1^{\circ}$ \\
\hline MichelObsF & $10.6^{\circ}$ & $5.4^{\circ}$ & $6.0^{\circ}$ & $5.8^{\circ}$ & $7.1^{\circ}$ & $7.7^{\circ}$ \\
\hline MichelEkf & $16.6^{\circ}$ & $7.0^{\circ}$ & $8.0^{\circ}$ & $8.9^{\circ}$ & $8.6^{\circ}$ & $10.1^{\circ}$ \\
\hline MichelEkfExtmag & $14.2^{\circ}$ & $8.9^{\circ}$ & $9.0^{\circ}$ & $5.5^{\circ}$ & $8.6^{\circ}$ & $9.2^{\circ}$ \\
\hline MichelEkfExtmagWt & $12.3^{\circ}$ & $6.3^{\circ}$ & $7.2^{\circ}$ & $5.3^{\circ}$ & $8.5^{\circ}$ & $8.7^{\circ}$ \\
\hline MichelEkfF & $10.8^{\circ}$ & $5.3^{\circ}$ & $5.5^{\circ}$ & $5.7^{\circ}$ & $10.3^{\circ}$ & $7.5^{\circ}$ \\
\hline
\end{tabular}

Table 10: Precision of attitude estimation according to typical motions with magnetic perturbations.

We observe that the two variants of our technique (MichelEkfF and MichelObsF) gives better precisions for all motions except for the back pocket motion in the case of MichelEkfF. MichelObsF thus stands out: it provides a significantly better precision during periods of magnetic perturbations even with high accelerations. We also notice that precision is improved regardless of the motion.

Figure 15 illustrates the relative improvements in precision brought by the respective components of our new technique presented in Section 7.1, in the case of yaw.

As a reminder, our both filters MichelObsF and MichelEkfF should provide the same precision than their native variant when magnetic perturbations are low. This is verified here (see Table 11), where MichelObsF has nearly the same results than Fourati and MichelEkfF has the same results than $E k f$.

\subsection{Limits and Perspectives}

We study motions which we consider reasonably representative of those commonly used with mobile applications. Obviously, there exist extreme cases of smartphone motions which make it very hard for any approach to provide a reliable attitude estimation. For example, the magnitude of the measured magnetic field in the presence of high magnetic perturbations can be equal to the magnitude of Earth's magnetic field. No approach is insensitive to this case, which might result in a drift of the attitude estimated.

Similarly, during long periods of magnetic perturbations, quality of attitude estimation depends mainly on the gyroscope drift. For example, if a user stays close to a heavy heater, the error in attitude estimation will progressively increase with time according to the gyroscope drift. 


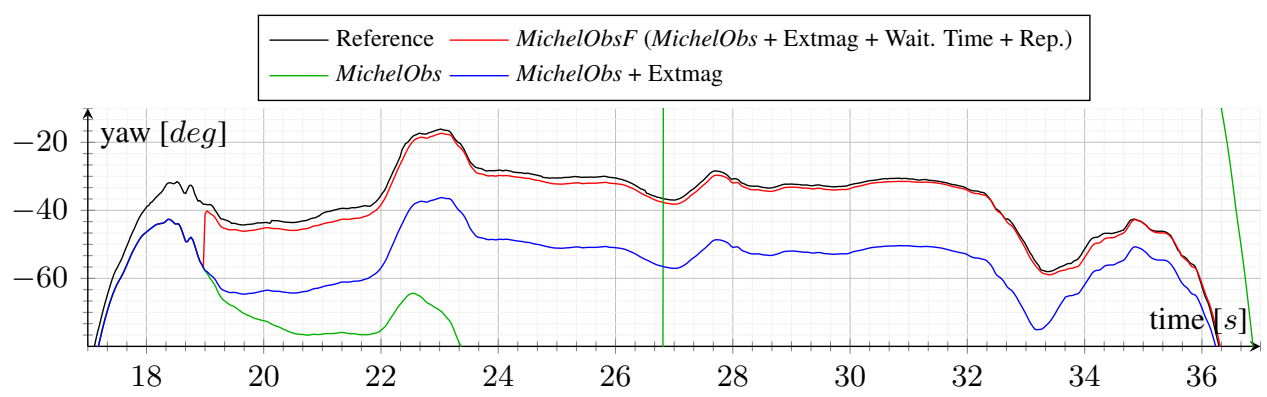

Figure 15: Sample run of the reprocessing technique (red) when a magnetic perturbation occurs, in comparison to ground truth (black) and earlier techniques.

\begin{tabular}{|c|c|c|c|c|c|c|c|c|}
\hline & $\frac{\alpha}{4}$ & 戠 & $\begin{array}{l}\stackrel{0}{\Xi} \\
\stackrel{\Xi}{0} \\
\stackrel{0}{0}\end{array}$ & 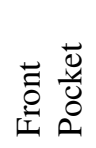 & 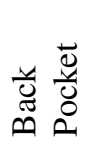 & 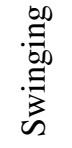 & 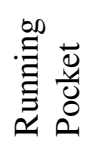 & 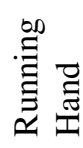 \\
\hline Fourati & $4.8^{\circ}$ & $4.0^{\circ}$ & $4.4^{\circ}$ & $4.6^{\circ}$ & $4.8^{\circ}$ & $5.3^{\circ}$ & $6.3^{\circ}$ & $6.6^{\circ}$ \\
\hline MichelObsF & $4.8^{\circ}$ & $3.9^{\circ}$ & $4.4^{\circ}$ & $4.6^{\circ}$ & $4.8^{\circ}$ & $5.3^{\circ}$ & $6.3^{\circ}$ & $6.6^{\circ}$ \\
\hline$E k f$ & $4.5^{\circ}$ & $4.0^{\circ}$ & $3.7^{\circ}$ & $4.6^{\circ}$ & $4.6^{\circ}$ & $5.9^{\circ}$ & $8.2^{\circ}$ & $16.8^{\circ}$ \\
\hline MichelEkfF & $4.5^{\circ}$ & $4.0^{\circ}$ & $3.7^{\circ}$ & $4.6^{\circ}$ & $4.6^{\circ}$ & $6.0^{\circ}$ & $8.2^{\circ}$ & $16.8^{\circ}$ \\
\hline
\end{tabular}

Table 11: Precision of attitude estimation according to typical motions without magnetic perturbations.

As a perspective for further work, it might be interesting to investigate the use of Renaudin et al. approach found in [5], involving the Quasi-Static-Field detector for magnetometer measurements during such long periods.

Finally, for the first corner case, it could be interesting to investigate how to use Eq. (3) in parallel with both detectors (Eq. (15) and Eq. (16).

\section{Attitude Estimation for Augmented Reality}

In this section, we focus on attitude estimation in the specific context of AR applications. These particular applications raise additional concerns. We will see that AR applications require a balance between precision of attitude estimation and the satisfaction of additional criteria. In this section, we give some insights on how to bring a much more immersive experience even if this translate into slight losses in precision.

Now, we will no longer try to find a filter which works with all motions (swinging, running...), we will look for a filter which can be used to enhance AR rendering. For the rest of the study, as Euler angles suffer from singularity [20] and this singularity is a problem when the smartphone is held in AR mode, we apply a rotation of $90^{\circ}$ around $\mathrm{x}$-axis then another rotation of $90^{\circ}$ around $\mathrm{z}$-axis to the results obtained. With this transformation, QAD is not affected, only Euler Angles are different from those we obtained in Section 6 The smartphone is now considered in "Camera landscape" frame, as shown in Figure 16. We now define: yaw, pitch and roll respectively as the rotations around $\mathrm{z}$-axis, $\mathrm{x}$-axis and $\mathrm{y}$-axis. 


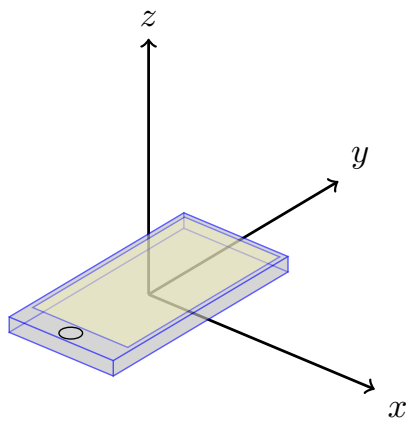

(a) Default frame

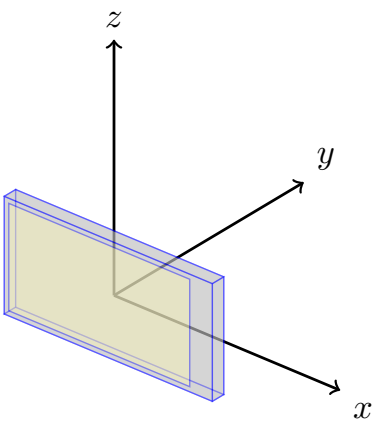

(b) Camera landscape frame

Figure 16: From default frame to camera landscape frame (rotation of $90^{\circ}$ around $\mathrm{x}$-axis then another rotation of $90^{\circ}$ around $\mathrm{z}$-axis)

In the benchmarks of Sections 6 and 7, during an AR motion, all filters exhibited a similar behavior when magnetic perturbations are low (see Section 6.4) and our proposed technique (with MichelObsF and MichelEkfF) outperforms other techniques during high magnetic perturbations (see Section 7). After implementing these algorithms in an AR application, we noticed two additional problems tied to the AR context and concerning all filters. We analyze them below and propose two approaches to enhance the AR experience of the user.

\subsection{Horizon-line tilted during magnetic perturbations}

During a magnetic perturbation, with all algorithms, except the one from Martin et al. [38], the horizon-line of virtual environment is tilted (see Figure 17). When the horizon line is tilted, in addition to the azimuth error (yaw-angle), point of interests will not be placed at the correct elevation (pitch-angle) and will suffer from a rotation (roll-angle).

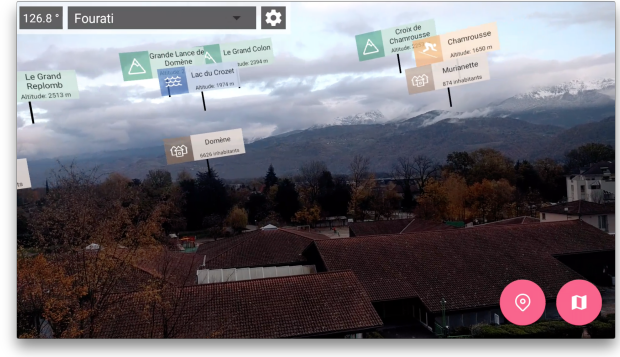

(a) Horizon-line is tilted (Fourati et al. [12])

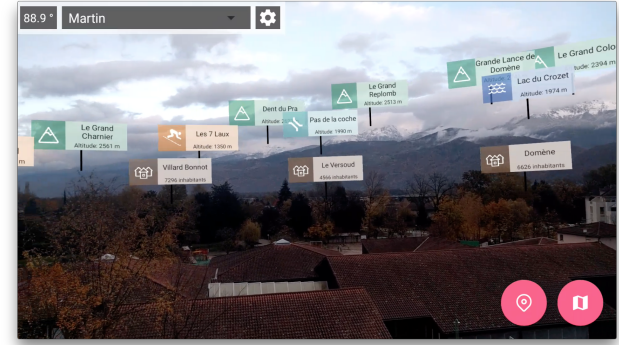

(b) Horizon-line is normal (Martin et al. [38])

Figure 17: A comparison bewteen a classical algorithm and an algorithm which compensate the tilted horizon-line.

Actually, in contrast to other algorithms, Martin's algorithm does not use magnetometer measurements on z-axis of Earth Frame (EF), i.e. pitch and roll should not be impacted during a magnetic perturbation. This is achieved by replacing the observation vector from magnetometer by the cross product of accelerometer and magnetometer. More specifically, let us consider the 
new frame: Magnetic Earth Frame (MF) where z-axis is pointing the sky, y-axis is pointing the Magnetic North and x-axis close the right-handed frame. The transformation from EF to MF is given by the following equation:

$$
M F=E F * \operatorname{rot}_{z}(d e c),
$$

where $d e c$ is the declination given by WMM (see Section 2.1.3)

Now, let us define ${ }^{M} \mathrm{C}$, the new observation vector in MF:

$$
\begin{aligned}
{ }^{M} \mathrm{acc} & =\left[\begin{array}{lll}
0 & 0 & g
\end{array}\right]^{T}, \\
{ }^{M} \mathrm{mag} & =\left[\begin{array}{lll}
0 & m_{y} & m_{z}
\end{array}\right]^{T}, \\
{ }^{M} \mathrm{C} & ={ }^{M} \text { acc } \wedge{ }^{M} \mathrm{mag}=\left[\begin{array}{lll}
-m_{y} * g & 0 & 0
\end{array}\right]^{T}
\end{aligned}
$$

$m_{z}$ is no longer used in the design of Martin's algorithm, as a consequence, magnetometer measurements will no longer be in conflict with accelerometer measurements and estimation of pitch and roll.

We then adapted this idea to two other algorithms that we studied in Section 4 Mahony and Fourati. The first resulting algorithm (MahonyAR) is a variant of Mahony. The second one (FouratiAR) is a variant of Fourati. We detail these algorithms below:

Algorithm - MahonyAR - $\left(\beta=0.2, K_{a}=1, K_{c}=0.5\right.$. $)$

$$
\begin{aligned}
& { }^{S} \mathrm{c}_{t}={ }^{S} \mathrm{acc}_{t} \wedge{ }^{S} \operatorname{mag}_{t} \\
& { }^{M} \mathrm{c}={ }^{M} \text { acc } \wedge{ }^{M} \mathrm{mag} \\
& S_{\mathrm{acc}_{q, t}}=\hat{q}_{t-1}^{-1} \otimes{ }^{E} \operatorname{acc}_{q, t} \otimes \hat{q}_{t-1} \\
& S_{\hat{\mathbf{c}}_{q, t}}=\hat{q}_{t-1}^{-1} \otimes{ }^{E} \mathrm{c}_{q, t} \otimes \hat{q}_{t-1} \\
& e=K_{a}\left[{ }^{S} \mathrm{acc}_{t} \times{ }^{S} \mathrm{acc}_{t}\right]+K_{c}\left[{ }^{S} \mathrm{c}_{t} \times{ }^{S} \hat{\mathrm{c}}_{t}\right] \\
& { }^{S} \hat{\operatorname{gyr}}_{q, t}={ }^{S} \operatorname{gyr}_{q, t}+k_{p} * e \text {, where: } \beta \text { is adjustable gain [37]. } \\
& \dot{\hat{q}}_{t}=\frac{1}{2} \hat{q}_{t-1} \otimes{ }^{S} \hat{g y r}_{q, t} \\
& \hat{q}_{t}=\hat{q}_{t-1}+\dot{\hat{q}}_{t} * \Delta t
\end{aligned}
$$


Algorithm - FouratiAR - $\left(\beta=0.3, K_{a}=2, K_{c}=1\right)$

$$
\begin{aligned}
& { }^{S} \mathrm{c}_{t}={ }^{S} \operatorname{acc}_{t} \wedge{ }^{S} \operatorname{mag}_{t} \\
& { }^{M} \mathrm{c}={ }^{M} \text { acc } \wedge{ }^{M} \mathrm{mag} \\
& S_{\mathrm{acc}_{q, t}}=\hat{q}_{t-1}^{-1} \otimes{ }^{M} \operatorname{acc}_{q} \otimes \hat{q}_{t-1} \\
& S_{\hat{\mathbf{c}}_{q, t}}=\hat{q}_{t-1}^{-1} \otimes{ }^{M} \mathrm{c}_{q} \otimes \hat{q}_{t-1} \\
& X=2\left[K_{a}\left[{ }^{S} \mathrm{acc}_{t} \times\right] \quad K_{c}\left[{ }^{S} \hat{\mathrm{c}}_{t} \times\right]\right]^{T} \\
& K=\left[X^{T} X+\lambda I_{3 \times 3}\right]^{-1} X^{T} \text {, with } \lambda=10^{-5} \\
& \hat{q}_{e, t}=\left[K\left[\begin{array}{c}
1 \\
{ }^{S} \operatorname{acc}_{t}-{ }^{S} \hat{a c c}_{t} \\
S_{\mathrm{c}_{t}}-{ }^{S} \hat{\mathbf{c}}_{t}
\end{array}\right]\right] \\
& \dot{\hat{q}}_{t}=\frac{1}{2} \hat{q}_{t-1} \otimes{ }^{S} \mathbf{g y r}_{q, t} \otimes \beta \hat{q}_{e, t} \\
& \hat{q}_{t}=\hat{q}_{t-1}+\dot{\hat{q}}_{t} * \Delta t
\end{aligned}
$$

Using the datasets recorded in the motion lab, we will now examine to which extent Martin's algorithm and the two new variants MahonyAR and FouratiAR are impacted on pitch and roll angles.

Table 12 shows the precision obtained with algorithms during AR motions in a highly perturbed magnetic environment (see Section 7.2).

\begin{tabular}{r|c|c|c|c|} 
& QAD & Yaw & Pitch & Roll \\
\hline black-box $^{*}$ & $29.0^{\circ}$ & $28.9^{\circ}$ & $\mathbf{1 . 1}^{\circ}$ & $\mathbf{1 . 2}^{\circ}$ \\
\hline Martin & $34.4^{\circ}$ & $34.1^{\circ}$ & $\mathbf{0 . 9}^{\circ}$ & $\mathbf{1 . 2}^{\circ}$ \\
\hline Fourati & $32.1^{\circ}$ & $31.5^{\circ}$ & $\mathbf{2 . 3}^{\circ}$ & $\mathbf{3 . 0}^{\circ}$ \\
\hline FouratiAR & $21.7^{\circ}$ & $21.3^{\circ}$ & $\mathbf{1 . 4}^{\circ}$ & $\mathbf{1 . 6}^{\circ}$ \\
\hline FouratiARF & $10.2^{\circ}$ & $9.8^{\circ}$ & $\mathbf{1 . 4}^{\circ}$ & $\mathbf{1 . 6}^{\circ}$ \\
\hline Mahony & $31.8^{\circ}$ & $28.9^{\circ}$ & $\mathbf{6 . 9}^{\circ}$ & $\mathbf{7 . 9}^{\circ}$ \\
\hline MahonyAR & $14.4^{\circ}$ & $14.1^{\circ}$ & $\mathbf{1 . 1}^{\circ}$ & $\mathbf{1 . 4}^{\circ}$ \\
\hline MahonyARF & $10.1^{\circ}$ & $9.8^{\circ}$ & $\mathbf{1 . 2}^{\circ}$ & $\mathbf{1 . 5}^{\circ}$ \\
\hline${ }^{*}$ black-box algorithms have been merged using average.
\end{tabular}

Table 12: Precision of attitude estimation for Augmented Reality motions with magnetic perturbations.

Algorithms which use Martin's et al. technique [38] yield better precision than others. For example, regarding pitch and roll angles, FouratiAR is twice accurate than its classical version. 
The same behavior is observed for MahonyAR algorithm which is 5 times more accurate than the Mahony version.

It is also possible to equip our filter with this technique in order to enhance overall results (MahonyARF and FouratiARF).

It should also be noticed that black-box algorithms have a good behavior in this specific context. It is likely that they use a similar technique.

\subsection{Importance of Filter Stability}

The second problem we encountered with some algorithms happens when the device is static: augmented points of interests might move or blink and consequently deteriorate user experience. That is not true for all of algorithms we tested.

For this purpose, we extended our benchmark with a part concerning stability. Stability is strongly related to the noise of sensors and to the trust in their measurements. For instance, when the accelerometer noise is higher than the noise of other sensors, if a filter mostly trusts accelerometer for estimation, attitude stability will mainly be impacted by accelerometer noise. Both sensors: magnetometer and accelerometer are known to exhibit a higher noise than the gyroscope [5, 41].

In order to investigate acceptable limits for stability, we asked the opinion of a panel of users.

\subsubsection{A User Experience of the Acceptable Stability Limit}

Precision error's STD cannot be used directly to know the stability of a filter. For this purpose, we used a moving STD with a window of $0.1 s$ which corresponds to the moving picture rate [48] observable by a user.

We designed a filter and created 7 variants from different set of parameters to obtain stabilities: from $0.09^{\circ} / s$ to $0.6^{\circ} / s$. The stability measurement makes sense only when filters assumptions are met (few magnetic perturbations and few external accelerations). Consequently, the survey has been conducted in an area with low magnetic perturbations and we designed an AR application with one PoI placed to the north. We asked the opinion of 20 people. For each filter's variant, the user is asked to choose between 3 propositions: filter's stability is not acceptable in any applications (X), filter's stability is acceptable within particular applications (O), filter's stability is excellent and well-suited for AR (V). Table 13 shows the raw results obtained during the survey.

We also report a summarized version of results in a graph-form to show the stability expected by a specific percentage of participants. We want to highlight two observations. The acceptable stability expected by $95 \%$ of the participants for an AR application is less than $0.28^{\circ} / \mathrm{s}$. For $95 \%$ of participants, a filter's stability is excellent when moving STD is less than $0.11^{\circ} \mathrm{s}$. 
Filter $\mathrm{n}^{\circ}$

\begin{tabular}{|c|c|c|c|c|c|c|}
$\# 1$ & $\# 2$ & $\# 3$ & $\# 4$ & $\# 5$ & $\# 6$ & $\# 7$ \\
\hline $\mathbf{0 . 0 9}$ & $\mathbf{0 . 1 1}$ & $\mathbf{0 . 1 9}$ & $\mathbf{0 . 2 8}$ & $\mathbf{0 . 3 9}$ & $\mathbf{0 . 4 6}$ & $\mathbf{0 . 6 0}$ \\
\hline V & V & V & O & O & X & X \\
\hline V & V & O & O & X & X & X \\
\hline V & V & V & O & O & X & X \\
\hline V & V & V & O & X & X & X \\
\hline V & V & V & O & X & X & X \\
\hline V & V & V & O & O & X & X \\
\hline V & V & V & O & X & X & X \\
\hline V & V & V & O & X & X & X \\
\hline V & V & O & O & X & X & X \\
\hline V & V & V & V & O & O & X \\
\hline V & V & V & V & O & O & X \\
\hline V & V & V & V & O & O & X \\
\hline V & V & V & O & O & O & X \\
\hline V & O & O & O & O & O & X \\
\hline V & V & O & O & O & X & X \\
\hline V & V & O & O & O & X & X \\
\hline V & V & O & O & X & X & X \\
\hline V & V & V & O & O & O & X \\
\hline V & V & V & O & X & X & X \\
\hline V & V & O & X & X & X & X \\
\hline & & & & & & \\
\hline V & & & \\
\hline
\end{tabular}

Table 13: User study on 7 filters stability. V: Excellent, O: Acceptable, X: Not recommended

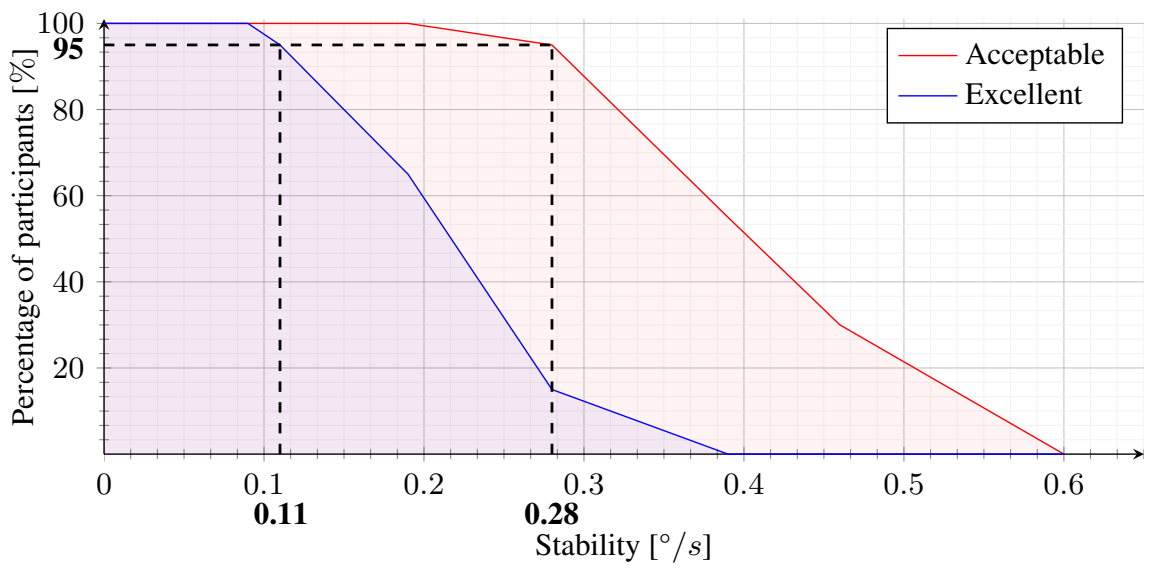




\subsubsection{Parameters Adjustment for a Balance Between Stability and Precision}

We now look for acceptable balances between stability and precision in the context of AR. In the previous sections, we evaluated algorithms using parameter values as recommended by their authors (Section 4). If authors did not provide instructions on setting parameter values, we chose them empirically. In the present section, we evaluate several sets of parameters for each filter in order to determine their feasibility envelope in terms of stability vs error. For the rest of the study, precision error of filters is shown in function of the stability.

Tests have been conducted with different sets of parameter values for each algorithm on a systematic basis (see Figure 18). Parameter values have been chosen empirically to cover a spectrum of possibilities and show the trade-off between the stability and the precision error. We recall below the set of parameters of each filter, and for each parameter we give the set of parameter values tested. We consider the cartesian product of all sets of parameter values. We indicate the size of the cartesian product (i.e. the number of configurations tested) next to each filter name.

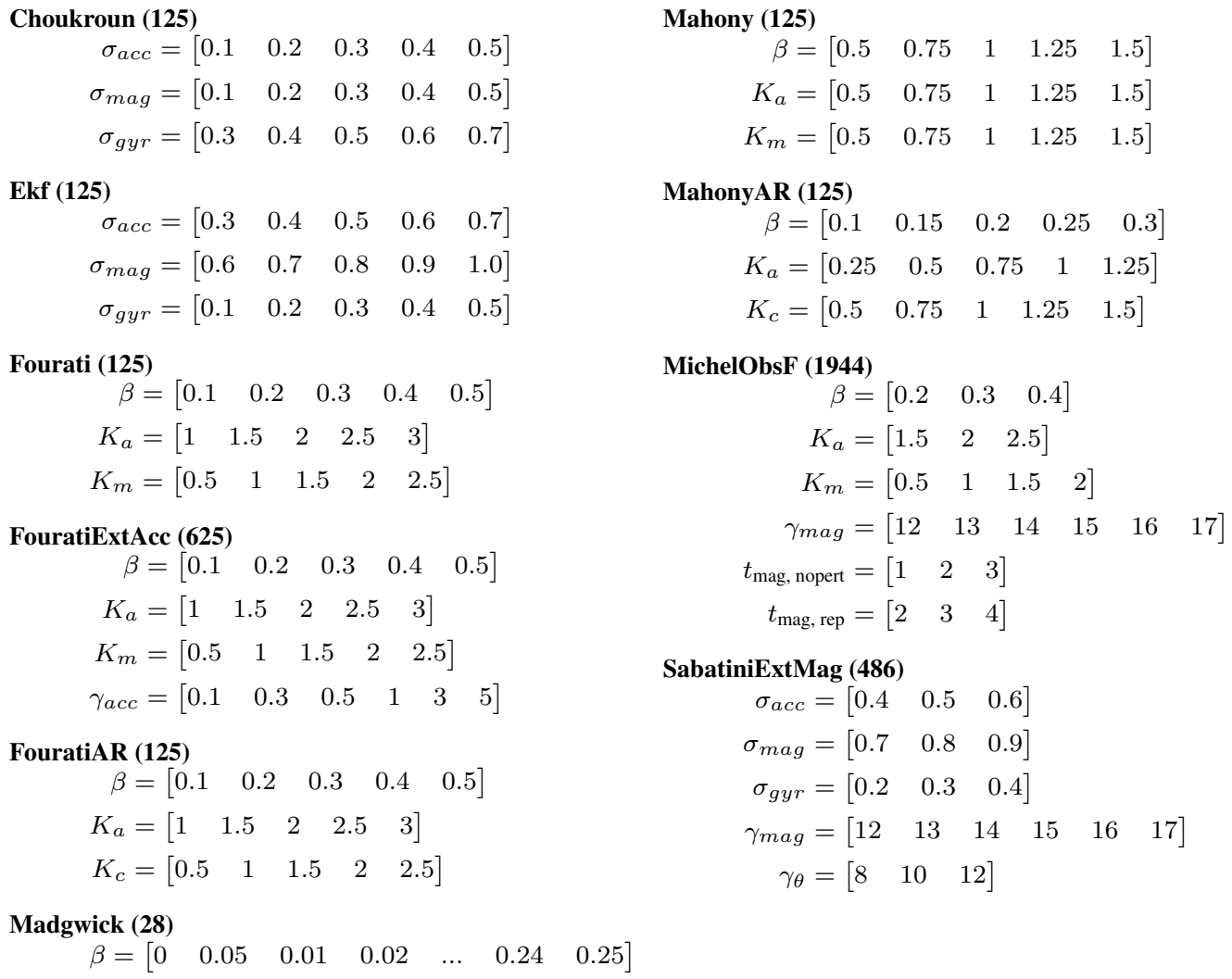

Figure 18: Sets of parameter values for each algorithm on a systematic basis.

For example, for MichelObsF we tested 1944 ways of setting initial parameter values, given 
by all the possible combinations of the values described above for each parameter.

We have set up an online too 7 to visualize the spectrum of possibilities for each algorithm. Figures 19 and 20 show the range of possibilities in terms of stability and precision error for a selection of algorithms during AR trials. Each dot of the graph corresponds to the couple

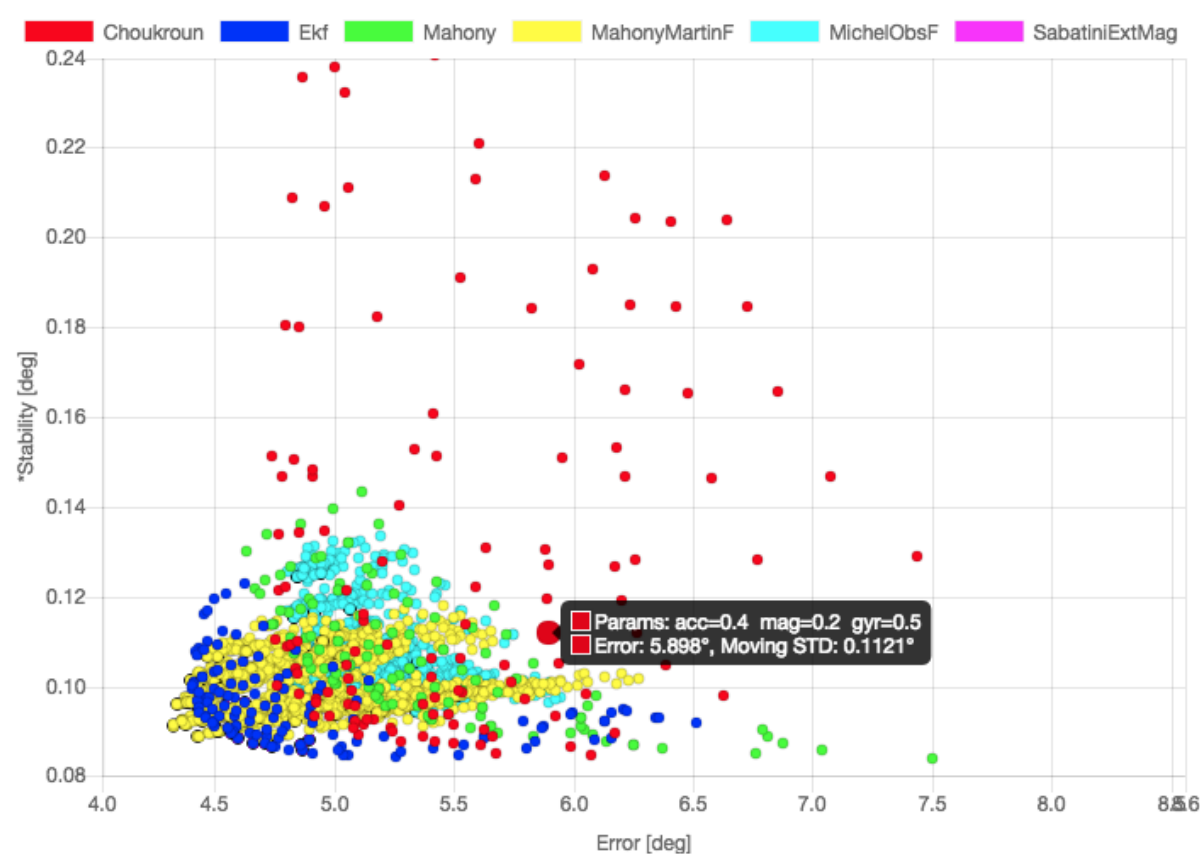

Figure 19: Spectrum of possibilities in terms of stability/precision in AR with few magnetic perturbations.

(precision error, stability) for one set of parameter values.

First of all, among the sets of parameters we chose for each algorithm, almost all of them provide a stability less than $0.28^{\circ} \%$ which is the stability expected by $95 \%$ of users for AR. Nevertheless, only few of them reach the excellent stability threshold $\left(0.11^{\circ} / s\right)$.

Concerning the algorithms precision, in the case of low magnetic perturbations (Fig. 19), we observed a common behavior for Kalman filters, whose best results are obtained when $\sigma_{\text {mag }} \approx$ $2 \sigma_{a c c}$ and $\sigma_{a c c} \approx 2 \sigma_{g y r}$. A similar observation holds with the weights of observers (instead of variances - thus with inverted ratios). Ratios found here between the sensors are directly related to sensor noises from the Allan variance [41].

In the case of high magnetic perturbations (Fig. 20), algorithms without detector exhibit a common behavior: their best results are obtained when $\sigma_{a c c}<\sigma_{m a g}$ and $\sigma_{g y r} \ll \sigma_{m a g}$. That behavior shows the impact of magnetic field measurements on the overall results. For algorithms with a magnetic perturbations detector, $\sigma_{g y r} \ll \sigma_{m a g}$ is also true, but $\sigma_{a c c} \approx 0.75 \sigma_{m a g}$.

To conclude about feasibility envelope, we observed that some filters offer more interesting envelopes than others, especially in the presence of magnetic perturbations (MichelObsF). Also, it is preferable to use a filter which deals with magnetic perturbations, this avoids to create a filter with adaptive parameters in function of the magnetic context.

7 http://tyrex.inria.fr/mobile/benchmarks-attitude/\#comparison-parameters 


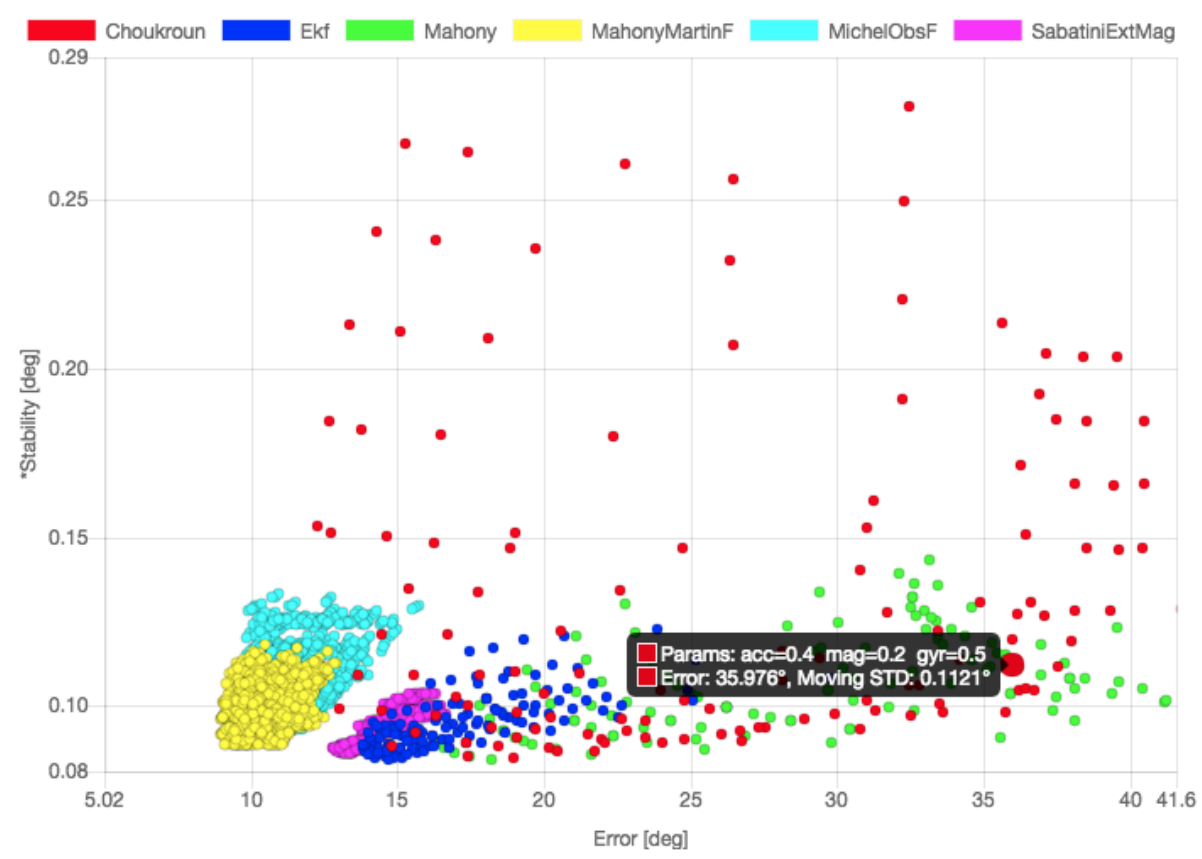

Figure 20: Spectrum of possibilities in terms of stability/precision in AR with high magnetic perturbations.

Moreover, our tool and experimental protocol allow us to confirm that parameter values chosen empirically in Section 4 are among those that yield the best results in this study.

To conclude on Augmented Reality, we would like to highlight that, while a given set of parameters can provide precise attitude estimation for the running motions, it might be unstable for AR. In Figure 21, we decided to illustrate this situation with the algorithm from Madgwick et al. where we make the $\beta$ parameter vary. During running motions, the best precision $\left(\approx 9^{\circ}\right)$ is reached when $\beta=0.24$. With the same parameter value, when the filter is used with $\mathrm{AR}$ motions, the precision is almost the best of the feasibility envelope $\left(\approx 5^{\circ}\right)$. However, the stability is particularly bad $\left(\approx .39^{\circ} / \mathrm{s}\right.$, which is higher than the stability expected by $95 \%$ of users for AR application; see Section 8.2.1.

\subsection{Remarks on Applicability}

The quality of a Geo Augmented Reality application cannot be stated just by studying the quality of the attitude estimation filter. It will also depend on the estimation of the user location and the distance between the device and the target to augment [49].

In [49], we proposed an evaluation method to calculate the average distance between a real and a virtual feature represented on the screen of the device. This system takes as input vectors of errors from the attitude estimation and positioning estimation approaches. We have shown that the distance between the feature and the user has a huge impact on rendering. In [49], we gave four use cases of AR applications, in which we applied our evaluation method.

Except for one very specific use case where the target to augment is very far away from the user (more than $1 \mathrm{~km}$ ), if we want to enhance the quality of augmented reality experience, 


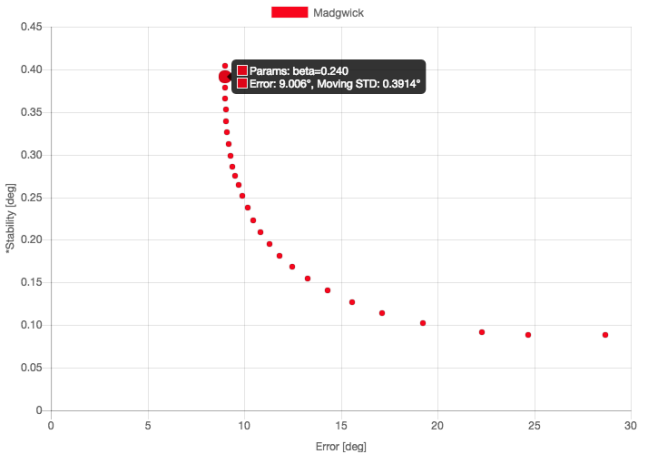

(a) Running motions

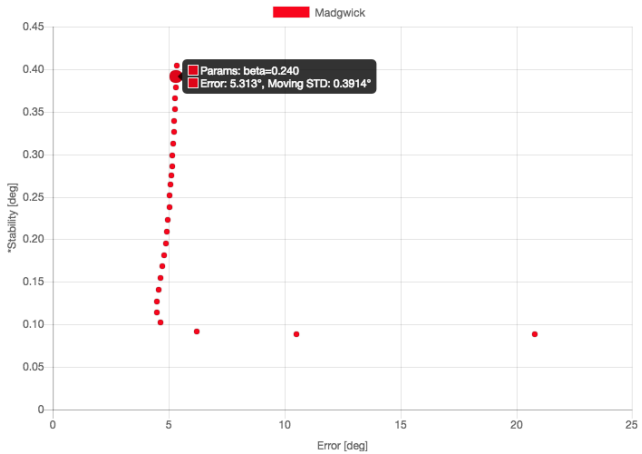

(b) AR motion

Figure 21: A comparison between AR motion and running motion for a same set of parameters with few magnetic perturbations.

efforts must now be made on the positioning estimation (for indoor and urban canyons) rather than attitude estimation.

\section{Conclusions}

We investigate the use of attitude estimation algorithms in the particular context of pedestrians using commodity smartphones. We propose a benchmark for evaluating and comparing the precision of attitude estimations during typical smartphone motions with and without magnetic perturbations. For the first time, our experiments shed light on the relative impacts of calibrations, parameters, noises, bias, motions, magnetic perturbations, and sampling rates when estimating attitude on smartphones. We go further in the study in the particular context of attitude estimation during augmented reality motions. An online tool based on the benchmarks has been released in order to help developers in choosing the right filter and appropriate parameter values in function of the expected motions, device, and magnetic perturbations. We also comment on lessons learned from our experiments for further research on the topic. In all cases, we recommend developers to use custom calibration and algorithms in replacement of those provided by smartphone's OS. Our algorithm "MichelObsF" provides significant gains in precision when estimating attitude in the presence of magnetic perturbations. In the absence of magnetic perturbations, it offers the same precision than the most precise algorithms. Furthermore, we tuned our algorithm with the best practices from the literature "MahonyARF" to enhance its precision and its stability when smartphone is used by an augmented reality application. As a perspective for future work, one might investigate how to adapt filter parameter values automatically in function of the recognized motion made with the smartphone.

\section{Acknowledgments}

This work has been partially supported by PERSYVAL-Lab (ANR11-LABX-0025), EquipEx KINOVIS (ANR-11-EQPX-0024). We thank J.F. Cuniberto for the smartphone handler, J. Zietsch and G. Dupraz-Canard for having walked for hours to record data and M. Razafimahazo for providing the iOS app. 


\section{Bibliography}

[1] G. Wahba, A least squares estimate of satellite attitude, SIAM review 7 (3) (1965) 409-409.

[2] W. GmbH, Wikitude URLWWW.wikitude.com

[3] Niantic, Inc., Ingress URL WWW.ingress.com

[4] Niantic, Inc., Pokemon GO URL Www.pokemongo.com

[5] V. Renaudin, C. Combettes, Magnetic, acceleration fields and gyroscope quaternion (MAGYQ)-based attitude estimation with smartphone sensors for indoor pedestrian navigation, Sensors 14 (12) (2014) 22864-22890.

[6] Google, Street view for mobile URL https://developers.google.com/streetview/android

[7] A. Brajdic, R. Harle, Walk detection and step counting on unconstrained smartphones, in: Proceedings of the 2013 ACM International Joint Conference on Pervasive and ubiquitous computing, ACM, 2013, pp. 225-234.

[8] L. Madden, Professional augmented reality browsers for smartphones: programming for junaio, layar and wikitude, John Wiley \& Sons, 2011.

[9] L. Ojeda, J. Borenstein, Flexnav: Fuzzy logi c expert rule-based position estimation for mobile robots on rugged terrain, in: Robotics and Automation, 2002. Proceedings. ICRA'02. IEEE International Conference on, Vol. 1, IEEE, 2002, pp. 317-322.

[10] E. J. Lefferts, F. L. Markley, M. D. Shuster, Kalman filtering for spacecraft attitude estimation, Journal of Guidance, Control, and Dynamics 5 (5) (1982) 417-429.

[11] M. Euston, P. Coote, R. Mahony, J. Kim, T. Hamel, A complementary filter for attitude estimation of a fixed-wing uav, in: Intelligent Robots and Systems, 2008. IROS 2008. IEEE/RSJ International Conference on, IEEE, 2008, pp. 340-345.

[12] H. Fourati, N. Manamanni, L. Afilal, Y. Handrich, A nonlinear filtering approach for the attitude and dynamic body acceleration estimation based on inertial and magnetic sensors: Bio-logging application, Sensors Journal, IEEE 11 (1) (2011) 233-244.

[13] I. Frosio, F. Pedersini, N. A. Borghese, Autocalibration of MEMS accelerometers, in: Advanced Mechatronics and MEMS Devices, Springer, 2013, pp. 53-88.

[14] U. (NGA), the U.K.'s Defence Geographic Centre (DGC), The world magnetic model, http://www. ngdc. noaa.gov/geomag/WMM [Online; accessed 17-July-2015] (2015).

[15] M. H. Afzal, V. Renaudin, G. Lachapelle, Magnetic field based heading estimation for pedestrian navigation environments, in: Indoor Positioning and Indoor Navigation (IPIN), 2011 International Conference on, IEEE, 2011, pp. 1-10.

[16] V. Renaudin, M. H. Afzal, G. Lachapelle, New method for magnetometers based orientation estimation, in: Position Location and Navigation Symposium (PLANS), IEEE, 2010, pp. 348-356.

[17] P. Bartz, Magnetic compass calibration, US Patent 4,698,912 (Oct. 13 1987)

[18] D. Sachs, Sensor fusion on android devices: A revolution in motion processing, [Video] https://www. youtube. com/watch? v=C7JQ7Rpwn2k [Online; accessed 9-April-2015] (2010).

[19] J. B. Kuipers, Quaternions and rotation sequences, Vol. 66, 1999.

[20] J. Diebel, Representing attitude: Euler angles, unit quaternions, and rotation vectors, Matrix 58 (2006) 15-16.

[21] H. D. Black, A passive system for determining the attitude of a satellite, AIAA journal 2 (7) (1964) 1350-1351.

[22] M. D. Shuster, S. Oh, Three-axis attitude determination from vector observations, Journal of Guidance, Control, and Dynamics 4 (1) (1981) 70-77.

[23] F. L. Markley, Attitude determination using vector observations and the singular value decomposition, The Journal of the Astronautical Sciences 36 (3) (1988) 245-258.

[24] T. Harada, H. Uchino, T. Mori, T. Sato, Portable absolute orientation estimation device with wireless network under accelerated situation, in: International Conference on Robotics and Automation, Vol. 2, IEEE, 2004, pp. 1412-1417.

[25] H. Rehbinder, X. Hu, Drift-free attitude estimation for accelerated rigid bodies, Automatica 40 (4) (2004) 653-659.

[26] D. Choukroun, I. Y. Bar-Itzhack, Y. Oshman, Novel quaternion kalman filter, Aerospace and Electronic Systems, IEEE Transactions on 42 (1) (2006) 174-190.

[27] J. K. Lee, E. J. Park, S. N. Robinovitch, Estimation of attitude and external acceleration using inertial sensor measurement during various dynamic conditions, IEEE transactions on instrumentation and measurement 61 (8) (2012) 2262-2273.

[28] R. G. Valenti, I. Dryanovski, J. Xiao, A linear kalman filter for marg orientation estimation using the algebraic quaternion algorithm, IEEE Transactions on Instrumentation and Measurement 65 (2) (2016) 467-481.

[29] J. L. Marins, X. Yun, E. R. Bachmann, R. B. McGhee, M. J. Zyda, An extended kalman filter for quaternion-based 
orientation estimation using marg sensors, in: Intelligent Robots and Systems, 2001. Proceedings. 2001 IEEE/RSJ International Conference on, Vol. 4, IEEE, 2001, pp. 2003-2011.

[30] A. Sabatini, Quaternion-based extended kalman filter for determining orientation by inertial and magnetic sensing, Biomedical Engineering, IEEE Transactions on 53 (7) (2006) 1346-1356.

[31] R. Zhu, D. Sun, Z. Zhou, D. Wang, A linear fusion algorithm for attitude determination using low cost mems-based sensors, Measurement 40 (3) (2007) 322-328.

[32] R. Munguia, A. Grau, Attitude and heading system based on ekf total state configuration, in: 2011 IEEE International Symposium on Industrial Electronics, IEEE, 2011, pp. 2147-2152.

[33] J. L. Crassidis, F. L. Markley, Unscented filtering for spacecraft attitude estimation, Journal of guidance, control, and dynamics 26 (4) (2003) 536-542.

[34] Y. S. Suh, Orientation estimation using a quaternion-based indirect kalman filter with adaptive estimation of external acceleration, IEEE Transactions on Instrumentation and Measurement 59 (12) (2010) 3296-3305.

[35] A. Makni, H. Fourati, A. Y. Kibangou, Adaptive kalman filter for mems-imu based attitude estimation under external acceleration and parsimonious use of gyroscopes, in: Control Conference (ECC), 2014 European, IEEE, 2014, pp. 1379-1384.

[36] Y. Oshman, A. Carmi, Attitude estimation from vector observations using a genetic-algorithm-embedded quaternion particle filter, Journal of Guidance, Control, and Dynamics 29 (4) (2006) 879-891.

[37] R. Mahony, T. Hamel, J.-M. Pflimlin, Nonlinear complementary filters on the special orthogonal group, Automatic Control, IEEE Transactions on 53 (5) (2008) 1203-1218.

[38] P. Martin, E. Salaün, Design and implementation of a low-cost observer-based attitude and heading reference system, Control Engineering Practice 18 (7) (2010) 712-722.

[39] S. O. Madgwick, A. J. Harrison, R. Vaidyanathan, Estimation of IMU and MARG orientation using a gradien descent algorithm, in: Rehabilitation Robotics (ICORR), 2011 IEEE International Conference on, IEEE, 2011, pp. $1-7$.

[40] F. L. Markley, D. Mortari, Quaternion attitude estimation using vector observations., Journal of the Astronautical Sciences 48 (2) (2000) 359-380.

[41] T. Michel, H. Fourati, P. Geneves, N. Layaïda, A comparative analysis of attitude estimation for pedestrian navigation with smartphones, in: Indoor Positioning and Indoor Navigation (IPIN), 2015 International Conference on, IEEE, 2015, pp. 1-10.

[42] T. Michel, P. Geneves, H. Fourati, N. Layaïda, On attitude estimation with smartphones, in: Pervasive Computing and Communications (PerCom), 2017 IEEE International Conference on, IEEE, 2017, pp. 267-275.

[43] W. Li, J. Wang, Effective adaptive kalman filter for mems-imu/magnetometers integrated attitude and heading reference systems, Journal of Navigation 66 (01) (2013) 99-113.

[44] E. R. Bachmann, X. Yun, C. W. Peterson, An investigation of the effects of magnetic variations on inertial/magnetic orientation sensors, in: Robotics and Automation, 2004. Proceedings. ICRA'04. 2004 IEEE International Conference on, Vol. 2, IEEE, 2004, pp. 1115-1122.

[45] K. Shoemake, Animating rotation with quaternion curves, in: ACM SIGGRAPH computer graphics, Vol. 19, ACM, 1985 , pp. 245-254.

[46] V. Renaudin, M. Susi, G. Lachapelle, Step length estimation using handheld inertial sensors, Sensors 12 (7) (2012) 8507-8525.

[47] D. Q. Huynh, Metrics for 3D rotations: Comparison and analysis, Journal of Mathematical Imaging and Vision 35 (2) (2009) 155-164

[48] S. K. Card, T. P. Moran, A. Newell, An engineering model of human performance, Ergonomics: Psychological mechanisms and models in ergonomics 1 (1986) 35

[49] T. Michel, On mobile augmented reality applications based on geolocation, Ph.D. thesis, Université Grenoble Alpes (2017). 